\title{
Magnetic field topology and chemical abundance distributions of the young, rapidly rotating, chemically peculiar star HR $5624^{\star}$
}

\author{
O. Kochukhov ${ }^{1}$, J. Silvester ${ }^{1}$, J. D. Bailey ${ }^{2}$, J. D. Landstreet ${ }^{3,4}$, and G. A. Wade ${ }^{5}$ \\ 1 Department of Physics and Astronomy, Uppsala University, Box 516, 75120 Uppsala, Sweden \\ e-mail: oleg.kochukhov@physics.uu.se \\ 2 Max Planck Insitut für Extraterrestrische Physik, Giessenbachstrasse 1, 85748 Garching, Germany \\ 3 Department of Physics and Astronomy, University of Western Ontario, London, Ontario, N6A 3K7, Canada \\ 4 Armagh Observatory, College Hill, Armagh, BT61 9DG, UK \\ 5 Department of Physics, Royal Military College of Canada, PO Box 17000, Stn Forces, Kingston, ON K7K 7B4, Canada
}

Received 3 April 2017 / Accepted 12 May 2017

\begin{abstract}
Context. The young, rapidly rotating Bp star HR 5624 (HD 133880) shows an unusually strong non-sinusoidal variability of its longitudinal magnetic field. This behaviour was previously interpreted as the signature of an exceptionally strong, quadrupole-dominated surface magnetic field geometry.

Aims. We studied the magnetic field structure and chemical abundance distributions of HR 5624 with the aim to verify the unusual quadrupolar nature of its magnetic field and to investigate correlations between the field topology and chemical spots.

Methods. We analysed high-resolution, time series Stokes parameter spectra of HR 5624 with the help of a magnetic Doppler imaging inversion code based on detailed polarised radiative transfer modelling of the line profiles.

Results. We refined the stellar parameters, revised the rotational period, and obtained new longitudinal magnetic field measurements. Our magnetic Doppler inversions reveal that the field structure of HR 5624 is considerably simpler and the field strength is much lower than proposed by previous studies. We find a maximum local field strength of $12 \mathrm{kG}$ and a mean field strength of $4 \mathrm{kG}$, which is about a factor of three weaker than predicted by quadrupolar field models. Our model implies that overall large-scale field topology of HR 5624 is better described as a distorted, asymmetric dipole rather than an axisymmetric quadrupole. The chemical abundance maps of $\mathrm{Mg}, \mathrm{Si}, \mathrm{Ti}, \mathrm{Cr}, \mathrm{Fe}$, and $\mathrm{Nd}$ obtained in our study are characterised by large-scale, high-contrast abundance patterns. These structures correlate weakly with the magnetic field geometry and, in particular, show no distinct element concentrations in the horizontal field regions predicted by theoretical atomic diffusion calculations.

Conclusions. We conclude that the surface magnetic field topology of HR 5624 is not as unusual as previously proposed. Considering these results together with other recent magnetic mapping analyses of early-type stars suggests that predominantly quadrupolar magnetic field topologies, invoked to be present in a significant number of stars, probably do not exist in real stars. This finding agrees with an outcome of the MHD simulations of fossil field evolution in stably stratified stellar interiors.
\end{abstract}

Key words. stars: atmospheres - stars: chemically peculiar - stars: magnetic field - starspots - stars: individual: HR 5624

\section{Introduction}

A small fraction of the upper-main sequence stars exhibit strong, globally-organised, stable surface magnetic fields (Donati \& Landstreet 2009). These fields, typically ranging between a few hundred $\mathrm{G}$ to several tens of $\mathrm{kG}$ in strength, are believed to be acquired by the stars at an earlier evolutionary stage rather than powered by a contemporary dynamo and are thus known as "fossil" fields. The phenomenon of surface fossil magnetism affects about 10 per cent of all main sequence stars in the interval between early $\mathrm{O}$ and early $\mathrm{F}$ spectral types (Wolff 1968; Donati \& Landstreet 2009; Wade et al. 2012; Fossati et al. 2015). The exact mechanisms responsible for the generation of these fields and explaining their relatively low incidence are yet to be conclusively established. Plausible hypotheses include an

\footnotetext{
* Based on observations collected at the European Southern Observatory, Chile (ESO programs 085.D-0296, 089.D-0383, 095.D-0194) and on observations obtained at the Canada-France-Hawaii Telescope (CFHT) which is operated by the National Research Council of Canada, the Institut National des Science de l'Univers of the Centre National de la Recherche Scientifique of France, and the University of Hawaii.
}

amplification of the magnetic flux during the gravitational collapse of protostellar clouds (Moss 2003), convective dynamos operating at the pre-main sequence evolutionary stage (Alecian 2014), or early stellar merger events (Schneider et al. 2016, and references therein). The presence of a strong magnetic field at the surfaces of early-type stars facilitates formation of inhomogeneities in the form of chemical abundance spots in B-F-type stars (Ap/Bp stars) or corotating circumstellar clouds in $\mathrm{O}$ and early B-type stars. These structures, in turn, give rise to conspicuous periodic photometric and spectroscopic variability.

Since the earliest studies of fossil magnetic fields (Stibbs 1950; Deutsch 1958) it has been evident that the majority of Ap/Bp stars show a single-wave, roughly sinusoidal periodic variation of the mean line-of-sight (longitudinal) magnetic field $\left\langle B_{z}\right\rangle$. This observation was interpreted in the framework of the phenomenological oblique rotator model (Stibbs 1950), which describes the stellar surface magnetic field topology as a frozenin dipole, inclined with respect to the rotational axis of the star. This model is still widely used for statistical studies of large stellar samples (e.g. Aurière et al. 2007; Hubrig et al. 2007) and as a starting guess for in-depth analyses of the field structure 
of individual stars. However, modelling $\left\langle B_{z}\right\rangle$ curves simultaneously with other integral magnetic observables (mean field modulus, crossover, mean quadratic field, broadband linear polarisation; see the comprehensive review by Mathys 2002) required higher-order multipolar field parameterisations in which quadrupolar terms often provided a significant, if not dominating, contribution (Landstreet \& Mathys 2000; Bagnulo et al. 2000, 2001, 2002). Moreover, several early-type magnetic stars, including HD 37776 (Thompson \& Landstreet 1985), HR 5624 (Landstreet 1990), $\tau$ Sco (Donati et al. 2006) and to a lesser extent HD 32633 (Silvester et al. 2012), HD 126515 (Mathys 2017) and HD 175362 (Mathys \& Hubrig 1997), show distinctly non-sinusoidal, double-wave $\left\langle B_{z}\right\rangle$ phase curves. In the literature, these observations are commonly considered as evidence of a field topology dominated by an axisymmetric quadrupolar component.

More recently, direct modelling of high-resolution Stokes profile time series observations with the help of magnetic Doppler imaging (MDI, Piskunov \& Kochukhov 2002; Kochukhov 2016) has provided new insight into fossil magnetic field geometries of early-type stars. Generally, the field topologies were found to be more diverse and occasionally much more complex than previously thought. Inversions of high-resolution Stokes parameter spectra, in particular those incorporating linear polarisation data, have suggested the presence of small-scale magnetic concentrations as well as toroidal fields superimposed onto a global, dipole-like magnetic topology (Kochukhov et al. 2004; Kochukhov \& Wade 2010; Silvester et al. 2014; Rusomarov et al. 2016). On the other hand, MDI of $\tau$ Sco and HD 37776, suspected to host dominant quadrupolar fields according to their non-sinusoidal $\left\langle B_{z}\right\rangle$ variation, have revealed highly structured, non-axisymmetric but weaker field geometries (Donati et al. 2006; Kochukhov et al. 2011; Kochukhov \& Wade 2016) than those inferred from the traditional low-order multipolar modelling of the integral magnetic observables. Thus, the question of the reality of strong, quadrupole-dominated fields in early-type stars is still unanswered. In particular, it remains unclear if these field models provide a faithful representation of the actual surface field structures occurring in a small number of stars or they are merely artefacts of parametric modelling limited to fitting the integral magnetic observables. The goal of this paper is to address this problem by investigating high-resolution time series Stokes parameter observations of HR 5624, which belongs to a group of rare Ap/Bp stars (along with HD 32633, HD 37776, HD 126515, HD 175362, and $\tau$ Sco) with anharmonic longitudinal field curves and hence presumably very unusual underlying magnetic field structures.

The late B-type star HR 5624 (HD 133880, HIP 74066, HR Lup) is a rapidly rotating, He-weak, Si-strong object. Despite its brightness $(V=5.8)$, it has seldom been analysed in detail due to its poor visibility from northern hemisphere observatories. This star is a member of the Upper Cen-Lup association and thus has a well-established age of about $16 \mathrm{Myr}$ (Landstreet et al. 2007). A strong magnetic field of HR 5624 was detected by Borra \& Landstreet (1975) and further investigated by Landstreet (1990) based on $\mathrm{H} \beta$ longitudinal magnetic field measurements. The non-sinusoidal character of the $\left\langle B_{z}\right\rangle$ phase variation led Landstreet (1990) to infer that the field topology is dominated by an axisymmetric quadrupole, which is at least three times stronger than the star's dipolar component. A detailed spectroscopic study of HR 5624 was presented by Bailey et al. (2012), who studied line profile variations, derived parameterised chemical abundance distributions, and refined the magnetic field topology model based on longitudinal field and Zeeman broadening measurements. Bailey et al. (2012) concluded that HR 5624 has an axisymmetric field structure described by a superposition of a $9.6 \mathrm{kG}$ dipole and $23.2 \mathrm{kG}$ quadrupole components, with the maximum local field strength exceeding $30 \mathrm{kG}$ and the mean field modulus reaching $20 \mathrm{kG}$. This exceptionally strong and unusually complex magnetic field must produce high-amplitude circular and linear polarisation signatures in spectral lines, making this star an ideal target for an MDI analysis.

In this paper we present a new investigation of the surface magnetic field structure and chemical spot distributions of HR 5624 using a superb spectropolarimetric data set collected for this star at the European Southern Observatory. The rest of the paper is organised as follows. Section 2 describes acquisition and reduction of the new spectropolarimetric observations of HR 5624. Section 3 discusses analysis methodology and presents main results, including a revision of the stellar parameters (Sect. 3.1), analysis of the mean polarisation profiles (Sect. 3.2) and mean longitudinal magnetic field (Sect. 3.3), qualitative diagnostic with the help of cumulative Stokes $V$ profiles (Sect. 3.4), and, finally, tomographic reconstruction of the magnetic and chemical abundance maps (Sect. 3.5). The results of our investigation are discussed in the context of theoretical magnetic field and atomic diffusion studies in Sect. 4 and summarised in Sect. 5.

\section{Spectropolarimetric observations}

The spectra of HR 5624 were obtained with the HARPSpol polarimeter (Snik et al. 2011; Piskunov et al. 2011) that feeds the HARPS spectrometer (Mayor et al. 2003) at the ESO $3.6 \mathrm{~m}$ telescope. This spectropolarimetric instrument allows one to obtain four Stokes parameter spectra at a resolving power of $\lambda / \Delta \lambda \approx 110000$ with a complete wavelength coverage of the $386-691 \mathrm{~nm}$ range apart from an $8 \mathrm{~nm}$ gap centred at $529 \mathrm{~nm}$. HARPSpol is equipped with two independent devices for circular and linear polarisation measurements. Each of them splits the incoming light into a pair of beams with orthogonal linear polarisation that are injected into fibres, dispersed by the echelle spectrograph and recorded simultaneously on a $4 \mathrm{~K} \times 4 \mathrm{~K} \mathrm{CCD}$ mosaic.

Each Stokes parameter observation with HARPSpol is comprised of a sequence of either two or four sub-exposures between which the retarder waveplates are rotated by $90^{\circ}$ (for Stokes $V$ observations) or by $45^{\circ}$ (for Stokes $Q U$ observations). This has an effect of exchanging positions of the orthogonally polarised spectra on the detector and enables application of a spectropolarimetric demodulation scheme known as the "ratio method" (Donati et al. 1997; Bagnulo et al. 2009). The two-exposure sequence yields a minimum data set for this demodulation procedure while four exposures provide a redundancy necessary for deriving the diagnostic null spectrum. To produce the latter, the sub-exposures are combined in such a way as to cancel the stellar polarisation signal, allowing one to assess spurious polarisation that might be present in the data.

The optimal extraction and wavelength calibration of the HARPSpol spectra of HR 5624 was carried out with the REDUCE code ${ }^{1}$ by Piskunov \& Valenti (2002). A custom set of IDL procedures was then used to perform continuum normalisation and spectropolarimetric demodulation. We refer the reader to the paper by Rusomarov et al. (2013) for an indepth discussion of the HARPSpol four Stokes parameter

\footnotetext{
http://wWw . astro.uu. se/ piskunov/RESEARCH/REDUCE/
} 
Table 1. Journal of HARPSpol observations of HR 5624.

\begin{tabular}{|c|c|c|c|c|c|c|c|c|c|}
\hline UT date & HJD & Phase & $\bar{S} S / N$ & Stokes & UT date & $\overline{\mathrm{HJD}}$ & Phase & $\overline{S S / N}$ & Stokes \\
\hline $03-05-2010$ & 2455319.7324 & 0.6996 & 226 & $I V$ & $12-05-2015$ & 2457154.7623 & 0.9414 & 293 & $I V$ \\
\hline 06-08-2012 & 2456145.5016 & 0.7650 & 103 & $I V$ & $12-05-2015$ & 2457154.7735 & 0.9542 & 297 & $I V$ \\
\hline $10-05-2015$ & 2457152.5257 & 0.3925 & 179 & $I V$ & $12-05-2015$ & 2457154.7847 & 0.9669 & 292 & $I V$ \\
\hline $10-05-2015$ & 2457152.5369 & 0.4053 & 117 & $I V$ & $12-05-2015$ & 2457154.7965 & 0.9804 & 295 & $I Q$ \\
\hline $10-05-2015$ & 2457152.5487 & 0.4188 & 110 & $I Q$ & $12-05-2015$ & 2457154.8077 & 0.9931 & 283 & $I Q$ \\
\hline $10-05-2015$ & 2457152.5599 & 0.4315 & 165 & $I Q$ & $12-05-2015$ & 2457154.8189 & 0.0059 & 283 & $I U$ \\
\hline $10-05-2015$ & 2457152.5711 & 0.4443 & 150 & $I U$ & $12-05-2015$ & 2457154.8300 & 0.0186 & 275 & $I U$ \\
\hline $10-05-2015$ & 2457152.5823 & 0.4570 & 132 & $I U$ & $12-05-2015$ & 2457154.8418 & 0.0320 & 251 & $I V$ \\
\hline $10-05-2015$ & 2457152.5951 & 0.4716 & 108 & $I V$ & $12-05-2015$ & 2457154.8530 & 0.0448 & 264 & $I V$ \\
\hline $10-05-2015$ & 2457152.6063 & 0.4843 & 117 & $I V$ & $12-05-2015$ & 2457154.8642 & 0.0575 & 238 & $I V$ \\
\hline $10-05-2015$ & 2457152.6184 & 0.4981 & 106 & $I Q$ & $12-05-2015$ & 2457154.8754 & 0.0703 & 220 & $I V$ \\
\hline $10-05-2015$ & 2457152.6295 & 0.5108 & 131 & $I Q$ & $12-05-2015$ & 2457154.8866 & 0.0830 & 215 & $I V$ \\
\hline $10-05-2015$ & 2457152.6408 & 0.5236 & 144 & $I U$ & $12-05-2015$ & 2457154.8978 & 0.0958 & 205 & $I V$ \\
\hline $10-05-2015$ & 2457152.6519 & 0.5364 & 144 & $I U$ & $13-05-2015$ & 2457156.4955 & 0.9166 & 203 & $I V$ \\
\hline $10-05-2015$ & 2457152.6643 & 0.5505 & 91 & $I V$ & $14-05-2015$ & 2457156.5067 & 0.9293 & 207 & $I V$ \\
\hline $10-05-2015$ & 2457152.6755 & 0.5632 & 52 & IV & $14-05-2015$ & 2457156.5190 & 0.9433 & 208 & $I Q$ \\
\hline $10-05-2015$ & 2457152.6883 & 0.5778 & 65 & $I Q$ & $14-05-2015$ & 2457156.5301 & 0.9561 & 212 & $I Q$ \\
\hline $10-05-2015$ & 2457152.6995 & 0.5906 & 70 & $I Q$ & $14-05-2015$ & 2457156.5474 & 0.9757 & 139 & $I$ \\
\hline $10-05-2015$ & 2457152.7107 & 0.6033 & 61 & $I U$ & $14-05-2015$ & 2457156.5590 & 0.9889 & 221 & $I V$ \\
\hline $10-05-2015$ & 2457152.7219 & 0.6161 & 31 & $I U$ & $14-05-2015$ & 2457156.5702 & 0.0017 & 210 & $I V$ \\
\hline $10-05-2015$ & 2457152.7336 & 0.6295 & 165 & $I V$ & $14-05-2015$ & 2457156.5814 & 0.0144 & 204 & $I V$ \\
\hline $10-05-2015$ & 2457152.7448 & 0.6422 & 213 & $I V$ & $14-05-2015$ & 2457156.5925 & 0.0272 & 200 & $I V$ \\
\hline $10-05-2015$ & 2457152.7567 & 0.6558 & 203 & $I Q$ & $14-05-2015$ & 2457156.6052 & 0.0417 & 260 & $I U$ \\
\hline $10-05-2015$ & 2457152.7679 & 0.6685 & 198 & $I Q$ & $14-05-2015$ & 2457156 & 0.0544 & 255 & $I U$ \\
\hline $10-05-2015$ & 2457152.7791 & 0.6813 & 201 & $I U$ & $14-05-2015$ & 2457156 & 0.0688 & 251 & $I Q$ \\
\hline $10-0$ & 2457152.7903 & 0.6941 & 191 & $I U$ & $14-05-$ & 2457156 & 0.0815 & 258 & $I Q$ \\
\hline $10-05-2015$ & 2457152.8022 & 0.7076 & 206 & $I V$ & $14-05-2015$ & 2457156 & 0.0943 & 250 & $I U$ \\
\hline $10-05-2015$ & 2457152.8134 & 0.7203 & 209 & $I V$ & $14-05-2015$ & 2457156 & 0.1070 & 249 & $I U$ \\
\hline $10-05-2015$ & 2457152.8265 & 0.7354 & 200 & $I Q$ & $14-05-2015$ & 2457156 & 0.1208 & 259 & $I V$ \\
\hline $10-05-2015$ & 2457152.8377 & 0.7481 & 210 & $I Q$ & $14-05-2015$ & 2457156.6859 & 0.1336 & 251 & $I V$ \\
\hline $10-05-2015$ & 2457152.8489 & 0.7609 & 165 & $I U$ & $14-05-2015$ & 2457156.6971 & 0.1463 & 280 & $I V$ \\
\hline $10-05-2015$ & 2457152.8601 & 0.7736 & 184 & $I U$ & $14-05-2015$ & 2457156.7083 & 0.1591 & 286 & $I V$ \\
\hline $10-05-2015$ & 2457152.8719 & 0.7871 & 171 & $I V$ & $14-05-2015$ & 2457156.7194 & 0.1718 & 291 & $I V$ \\
\hline $10-05-2015$ & 2457152.8831 & 0.7998 & 164 & $I V$ & $14-05-2015$ & 2457156.7306 & 0.1845 & 280 & $I V$ \\
\hline $12-05-2015$ & 2457154.5078 & 0.6514 & 153 & $I V$ & $14-05-2015$ & 2457156.7433 & 0.1990 & 256 & $I Q$ \\
\hline $12-05-2015$ & 2457154.5190 & 0.6641 & 170 & $I V$ & $14-05-2015$ & 2457156.7545 & 0.2117 & 282 & $I Q$ \\
\hline $12-05-2015$ & 2457154.5302 & 0.6769 & 133 & $I V$ & $14-05-2015$ & 2457156.7671 & 0.2261 & 259 & IV \\
\hline $12-05-2015$ & 2457154.5414 & 0.6896 & 85 & $I V$ & $14-05-2015$ & 2457156.7783 & 0.2388 & 233 & $I V$ \\
\hline $12-05-2015$ & 2457154.5534 & 0.7033 & 57 & $I Q$ & $14-05-2015$ & 2457156.7894 & 0.2516 & 226 & $I V$ \\
\hline $12-05-2015$ & 2457154.6550 & 0.8191 & 255 & $I Q$ & $14-05-2015$ & 2457156.8006 & 0.2643 & 207 & $I V$ \\
\hline $12-05-2015$ & 2457154.6662 & 0.8318 & 271 & $I Q$ & 14-05-2015 & 2457156.8118 & 0.2771 & 178 & $I V$ \\
\hline $12-05-2015$ & 2457154.6774 & 0.8446 & 285 & $I U$ & $14-05-2015$ & 2457156.8230 & 0.2898 & 184 & $I V$ \\
\hline $12-05-2015$ & 2457154.6858 & 0.8542 & 201 & $I$ & $14-05-2015$ & 2457156.8363 & 0.3049 & 156 & $I U$ \\
\hline $12-05-2015$ & 2457154.7058 & 0.8771 & 281 & $I U$ & $14-05-2015$ & 2457156.8474 & 0.3177 & 117 & $I U$ \\
\hline $12-05-2015$ & 2457154.7170 & 0.8898 & 296 & $I U$ & $14-05-2015$ & 2457156.8608 & 0.3329 & 170 & $I V$ \\
\hline $12-05-2015$ & 2457154.7288 & 0.9032 & 297 & $I V$ & $14-05-2015$ & 2457156.8720 & 0.3457 & 129 & $I V$ \\
\hline $12-05-2015$ & 2457154.7400 & 0.9159 & 288 & IV & $14-05-2015$ & 2457156.8832 & 0.3584 & 101 & $I V$ \\
\hline $12-05-2015$ & 2457154.7511 & 0.9287 & 282 & $I V$ & $14-05-2015$ & 2457156.8944 & 0.3711 & 56 & $I V$ \\
\hline
\end{tabular}

Notes. The columns give the UT date at mid-observation, the corresponding heliocentric Julian date (HJD), rotational phase for $P_{\text {rot }}=0.877483 \mathrm{~d}$, the median signal-to-noise ratio per $0.8 \mathrm{~km} \mathrm{~s}^{-1}$ pixel in the $490-500 \mathrm{~nm}$ wavelength region, and the Stokes parameters obtained, from left to right, respectively. Observations listed here were determined from two sub-exposures with the exception of the two Stokes $I$ spectra (phases 0.8542 and 0.9757), which were derived from single exposures.

observing procedures and detailed description of our data reduction pipeline.

The observations of HR 5624 were performed on the nights of May 10, 12, and 14, 2015. Initially, we planned to secure a complete rotational phase coverage in all four Stokes parameters and therefore obtained on the first observing night six Stokes $V$, five Stokes $Q$, and five Stokes $U$ observations, each consisting of four $450 \mathrm{~s}$ sub-exposures. However, having examined these data, we found that HR 5624 exhibits a significantly lower polarisation amplitude than predicted by the previous field geometry models of this star, rendering the signal-to-noise ratio $(S / N)$ in the linear polarisation profiles insufficient for detailed modelling. Consequently, we modified the observing strategy for the remainder of the observing run, putting more emphasis on the Stokes $V$ observations. The final set of Stokes parameter spectra was derived from pairs of sub-exposures in order to improve the phase resolution. Analysis of the diagnostic null profiles obtained from four sub-exposure sequences showed no evidence of a spurious polarisation in any of the HARPSpol Stokes parameter observations.

The resulting HARPSpol data set analysed in this paper includes 52 Stokes $V$ spectra $(50$ observations from our 2015 observing run plus two archival circular polarisation observations obtained in 2010 and 2012) as well as 21 Stokes $Q, 21$ Stokes $U$, 
and 96 intensity (Stokes $I$ ) measurements. Table 1 provides information on each of these observations, including mid-exposure UT date, heliocentric Julian date, rotational phase, and representative $S / N$. We note that two of the intensity spectra listed in this table correspond to interrupted polarimetric sequences and therefore do not have associated polarisation spectra.

In addition to the HARPSpol data, we have used 14 ESPaDOnS Stokes $V$ spectra of HR 5624 obtained from 2010 to 2012. These data, retrieved from the CFHT Science Archive ${ }^{2}$, were reduced by the UPENA pipeline running the LIBRE ESPRIT software (an updated version of the code described by Donati et al. 1997). The ESPaDOnS spectra cover the 370$1050 \mathrm{~nm}$ wavelength interval at a resolution of about 65000 . The first three of these spectra were analysed by Bailey et al. (2012). Considering that the HARPSpol data have a superior resolution and adequate phase coverage, we have used the ESPaDOnS spectra of HR 5624 only for the purpose of deriving additional longitudinal field measurements (see Sect. 3.3).

\section{Analysis and results}

\subsection{Stellar parameters}

Several determinations of the atmospheric and fundamental parameters of HR 5624 are available in the literature. The stellar effective temperature was determined by Kochukhov \& Bagnulo (2006, $12000 \pm 400 \mathrm{~K})$, Landstreet et al. (2007, $12000 \pm 400 \mathrm{~K})$, and Netopil et al. $(2008,11930 \pm 210 \mathrm{~K})$ based on the Geneva and Strömgren photometric calibrations appropriate for magnetic, chemically peculiar stars. On the other hand, Bailey et al. (2012) refrained from applying an Ap-star correction to their photometric temperature estimate and adopted $T_{\text {eff }}=13000 \pm$ $600 \mathrm{~K}$.

Based on the comparison with stellar evolutionary models and HIPPARCos parallax, Kochukhov \& Bagnulo (2006) established $\log L / L_{\odot}=2.07 \pm 0.10, R / R_{\odot}=2.51 \pm 0.33$, and $M / M_{\odot}=$ $3.10 \pm 0.12$. Landstreet et al. (2007) used the fact that HR 5624 is a member of the Upper Centaurus Lupus association and therefore has a well-established age of $\log t=7.2 \pm 0.1 \mathrm{yr}$. These authors obtained $\log L / L_{\odot}=2.10 \pm 0.10, R / R_{\odot}=2.60 \pm 0.38$, and $M / M_{\odot}=3.20 \pm 0.15$, consistent with a young star that has completed about 5 per cent of its main sequence lifetime.

In this paper we took advantage of the high-resolution spectra around hydrogen Balmer lines to test different sets of atmospheric parameters. We have computed a grid of LLMODELS (Shulyak et al. 2004) atmospheres using the average abundances reported by Bailey et al. (2012) and adopting a magnetic field of $5 \mathrm{kG}$ to roughly account for the Zeeman desaturation of spectral lines. The hydrogen $\mathrm{H} \beta$ and $\mathrm{H} \gamma$ lines, computed for each model atmosphere in the grid using the SYNMAST spectrum synthesis code (Kochukhov et al. 2010), were compared with the average HARPSpol spectra to establish the best-fitting $T_{\text {eff }}$ and $\log g$. As demonstrated by Fig. $1, T_{\text {eff }}=12000 \mathrm{~K}$ and $\log g=4.15$ provide a better description of the observations compared to $T_{\text {eff }}=$ $13000 \mathrm{~K}$ and $\log g=4.34$ used by Bailey et al. (2012). Based on this comparison, we adopted the former set of atmospheric parameters for further analysis. We note that our $\log g$ established from fitting the hydrogen Balmer lines is in good agreement with the surface gravity of $\log g=4.11-4.13$ that can be inferred from the stellar evolution analyses by Kochukhov \& Bagnulo (2006) and Landstreet et al. (2007).

\footnotetext{
2 http://www.cadc-ccda.hia-iha.nrc-cnrc.gc.ca/en/ cfht/
}
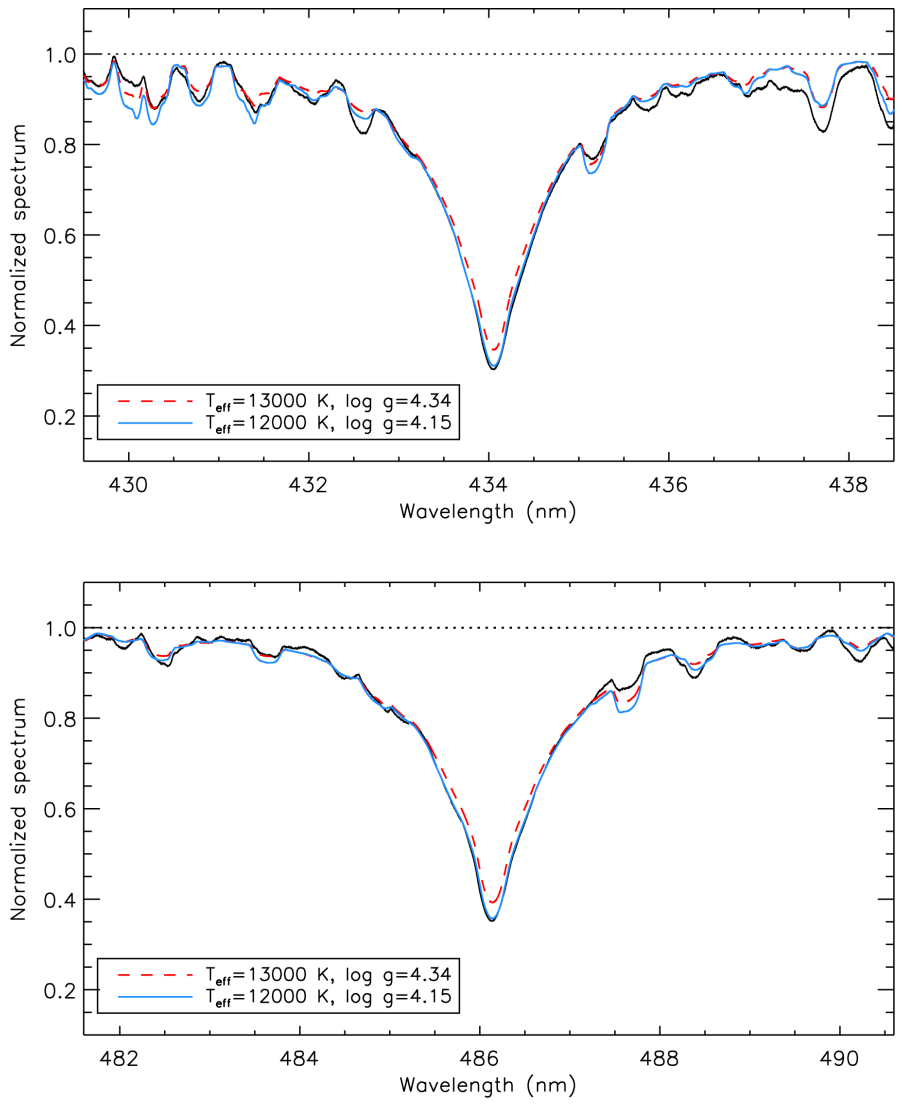

Fig. 1. Comparison of the mean observed hydrogen $\mathrm{H} \gamma$ and $\mathrm{H} \beta$ profiles (black histograms) with the synthetic spectra calculated for $T_{\text {eff }}=13000 \mathrm{~K}, \log g=4.34$ (dashed red line) and $T_{\text {eff }}=12000 \mathrm{~K}$, $\log g=4.15$ (solid blue line).

\subsection{Least-squares deconvolved profiles}

The Stokes $V$ profiles of strong metal lines in the spectrum of HR 5624 exhibit distinct circular polarisation signatures. However, their relatively low $S / N$ and often severe blending renders individual lines unsuitable for detailed modelling. No linear polarisation signatures could be detected in any of the individual spectral lines. Considering these circumstances, we applied the least-squares deconvolution (LSD) multi-line method (Donati et al. 1997), implemented as described by Kochukhov et al. (2010), in order to obtain high-quality mean Stokes profiles for all metal lines and for subsets of a few selected chemical elements. The line parameters required for building an LSD mask were obtained from the VALD3 database (Ryabchikova et al. 2015) using the $T_{\text {eff }}=12000 \mathrm{~K}, \log g=$ 4.15 model atmosphere described above and the mean abundances from Bailey et al. (2012). We then retained lines deeper than 5 per cent of the continuum (before any macroscopic broadening) and excluded all lines located in the wings of the hydrogen Balmer lines or in the regions of significant telluric absorption. Four sets of Stokes IQUV LSD profiles were derived: i) using all 1916 available metal lines; ii) using 1127 Fe lines; iii) using $275 \mathrm{Cr}$ lines; and iv) using $167 \mathrm{Si}$ lines. The LSD line weights for the complete metal line mask were normalised using a mean wavelength $\lambda_{0}=523 \mathrm{~nm}$, Landé factor $z_{0}=1.18$, and depth $d_{0}=0.18$. A common normalisation with $\lambda_{0}=518 \mathrm{~nm}$, $z_{0}=1.20, d_{0}=0.17$ was adopted for the Fe, Cr, and Si LSD profiles. As discussed by Kochukhov et al. (2010), the choice of LSD line weight normalisation is irrelevant as long as it is applied consistently in derivation of LSD profiles and in their 

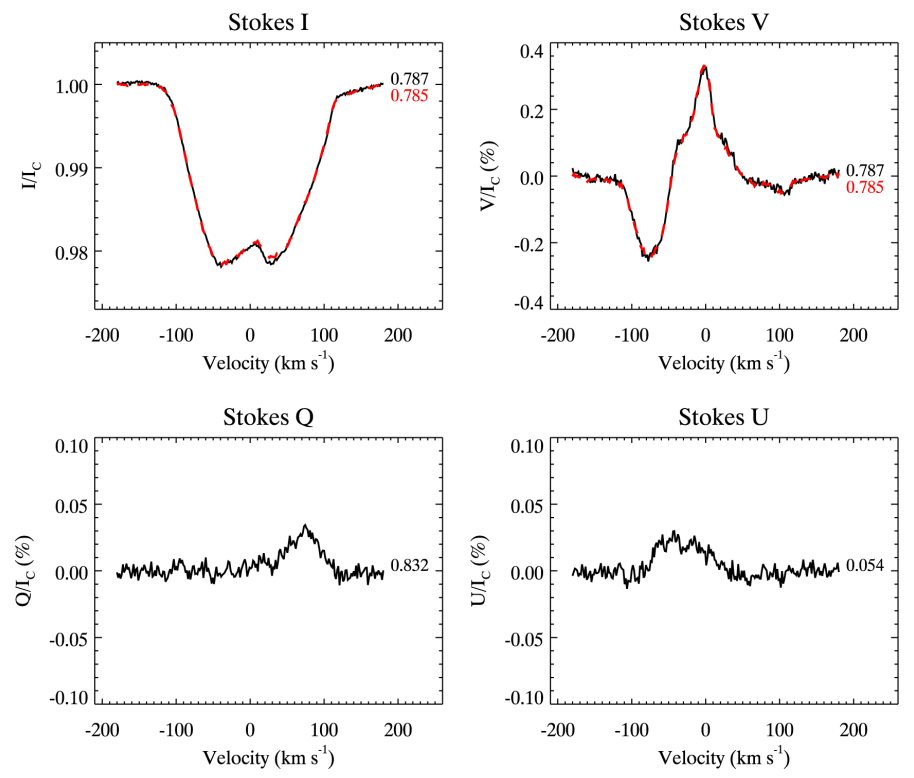

Fig. 2. Example of the four Stokes parameter LSD profiles derived for HR 5624 using a complete metal line mask. The top panels compare the Stokes $I$ and $V$ HARPSpol profiles (solid lines) with the ESPaDOnS LSD profiles (dashed lines) obtained at close rotational phases. The bottom panels show examples of some of the strongest Stokes $Q$ and $U$ HARPSpol LSD profiles.

interpretation. The same line lists and deconvolution procedures were applied to both HARPSpol and ESPaDOnS spectra; the LSD velocity step was chosen to be $1.6 \mathrm{~km} \mathrm{~s}^{-1}$ for HARPSpol and $2.6 \mathrm{~km} \mathrm{~s}^{-1}$ for the ESPaDOnS data, respectively. For consistency, the LSD analysis of the ESPaDOnS spectra was restricted to the HARPS wavelength range.

Figure 2 shows an example of the four Stokes parameter LSD profiles derived from the HARPSpol observations. For comparison, we also present in the upper panels of this figure the Stokes $I$ and $V$ LSD profiles corresponding to a nearby rotational phase observed by ESPaDOnS. The circular polarisation signal is detected with a high $S / N$, which is also the case for the Fe, $\mathrm{Si}$, and Cr LSD profiles. On the other hand, the Stokes $Q$ and $U$ signals are detected at a relatively low $S / N$ only in the LSD profiles corresponding to the complete metal line mask. The bottom panels of Fig. 2 display some of the strongest linear polarisation mean line signatures detected in the HARPSpol data.

A dense phase coverage secured by our HARPSpol observations, combined with plenty of sharp details evident in the Stokes $I$ and $V$ LSD profiles, enable an accurate determination of the stellar rotational period. Previous period determinations for HR 5624 were summarised by Bailey et al. (2012), who converged on the value of $0.877476 \pm 0.000009 \mathrm{~d}$. Considering phasing of the 2010 archival HARPSpol spectrum relative to our 2015 data set, we find a slight offset (Fig. 3, upper panels). A revised period of $0.877483 \mathrm{~d}$, which is only $0.8 \sigma$ longer than the value preferred by Bailey et al. (2012), yields a smooth variation of the Stokes $I$ and $V$ profiles with a minimum phase dispersion (Fig. 3, lower panels). The same period also improves the phasing of the 2010-2012 ESPaDOnS spectra relative to the 2015 HARPSpol data. Therefore, we adopted this period and the reference Julian date 2445472.000 (Bailey et al. 2012) corresponding to the minimum photometric brightness for the MDI modelling presented below.
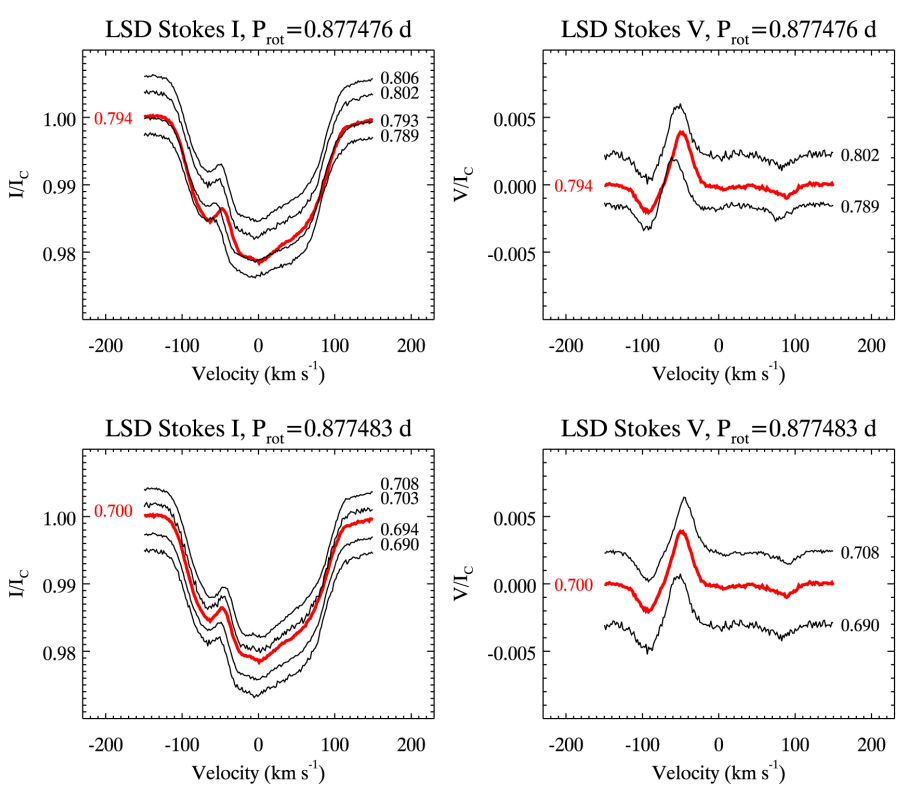

Fig. 3. Relative phasing of the LSD Stokes $I$ (left) and $V$ (right) profiles corresponding to the archival HARPSpol observation of HR 5624 obtained in 2010 (thick, light/red curves) and observations collected in 2015 (thin, dark/black curves) for the rotational periods 0.877476 (top) and 0.877483 days (bottom). Profiles are shifted vertically according to the rotational phases, which are indicated to the right of the profiles for the 2015 data and to the left for the 2010 spectrum.

\subsection{Mean longitudinal magnetic field}

The mean longitudinal magnetic field $\left\langle B_{z}\right\rangle$ measures the diskaveraged line-of-sight magnetic field component, weighted by the local continuum brightness and line strength. This observable is commonly used for characterising magnetic fields of $\mathrm{Ap} / \mathrm{Bp}$ stars. The non-sinusoidal variation of $\left\langle B_{z}\right\rangle$ in HR 5624 was considered as the basic primary evidence of a quadrupole-dominated magnetic field topology in this star (Landstreet 1990).

We have inferred the mean longitudinal magnetic field of HR 5624 from the Stokes $I$ and $V$ LSD profiles corresponding to the complete metal line mask. These $\left\langle B_{z}\right\rangle$ measurements were derived by calculating the first moment of the Stokes $V$ profile, normalised by the equivalent width of the corresponding Stokes $I$ profile and scaled by the appropriate mean wavelength and Landé factor (Kochukhov et al. 2010). The numerical integration was carried out in the velocity range between -120 and $+125 \mathrm{~km} \mathrm{~s}^{-1}$ and was applied consistently to both HARPSpol and ESPaDOnS data. The resulting $\left\langle B_{z}\right\rangle$ values, summarised in Table 2, indicate variation between about -3.9 and $+2.3 \mathrm{kG}$. The median error bar of our measurements is $47 \mathrm{G}$.

The phase variation of the HARPSpol and ESPaDOnS $\left\langle B_{z}\right\rangle$ measurements agree very well. These data are also compatible, despite a difference in measurement methodology, with the hydrogen line photopolarimetric longitudinal field measurements collected by Borra \& Landstreet (1975) and Landstreet (1990) provided that $P_{\text {rot }}=0.877476 \mathrm{~d}$ is used. However, for our preferred $P_{\text {rot }}=0.877483 \mathrm{~d}$ there is a 0.07 phase offset between the photopolarimetric data from 1974-1988 and the new spectropolarimetric measurements obtained from 2010-2015. This offset cannot be removed by postulating a simple linear period increase corresponding to the canonical rotational braking (Ud-Doula et al. 2009). Instead, a step-like or continuous period decrease, comparable to the one experienced by CU Vir 
Table 2. Mean longitudinal magnetic field of HR 5624.

\begin{tabular}{crc|crc|crc}
\hline \hline HJD & \multicolumn{1}{c|}{$\left\langle B_{z}\right\rangle(\mathrm{G})$} & Inst & HJD & \multicolumn{1}{c}{$\left\langle B_{z}\right\rangle(\mathrm{G})$} & Inst & HJD & $\left\langle B_{z}\right\rangle(\mathrm{G})$ & Inst \\
\hline 2455319.7324 & $863 \pm 46$ & $\mathrm{H}$ & 2457154.5190 & $1548 \pm 59$ & $\mathrm{H}$ & 2457156.5067 & $-3890 \pm 42$ & $\mathrm{H}$ \\
2456145.5016 & $-1266 \pm 89$ & $\mathrm{H}$ & 2457154.5302 & $1335 \pm 74$ & $\mathrm{H}$ & 2457156.5590 & $-3590 \pm 41$ & $\mathrm{H}$ \\
2457152.8134 & $112 \pm 48$ & $\mathrm{H}$ & 2457154.5414 & $1023 \pm 111$ & $\mathrm{H}$ & 2457156.5702 & $-3609 \pm 43$ & $\mathrm{H}$ \\
2457152.8719 & $-1986 \pm 54$ & $\mathrm{H}$ & 2457154.7288 & $-3922 \pm 33$ & $\mathrm{H}$ & 2457156.5814 & $-3595 \pm 45$ & $\mathrm{H}$ \\
2457152.8831 & $-2459 \pm 56$ & $\mathrm{H}$ & 2457154.7400 & $-3902 \pm 34$ & $\mathrm{H}$ & 2457156.5925 & $-3355 \pm 46$ & $\mathrm{H}$ \\
2457152.5257 & $1809 \pm 69$ & $\mathrm{H}$ & 2457154.7511 & $-3894 \pm 34$ & $\mathrm{H}$ & 2457156.6747 & $-1279 \pm 40$ & $\mathrm{H}$ \\
2457152.5369 & $1790 \pm 105$ & $\mathrm{H}$ & 2457154.7623 & $-3782 \pm 33$ & $\mathrm{H}$ & 2457156.6859 & $-937 \pm 41$ & $\mathrm{H}$ \\
2457152.5951 & $1698 \pm 113$ & $\mathrm{H}$ & 2457154.7735 & $-3742 \pm 33$ & $\mathrm{H}$ & 2457156.6971 & $-521 \pm 38$ & $\mathrm{H}$ \\
2457152.6063 & $1586 \pm 104$ & $\mathrm{H}$ & 2457156.7083 & $-173 \pm 38$ & $\mathrm{H}$ & 2455411.7310 & $2018 \pm 42$ & $\mathrm{E}$ \\
2457152.6643 & $1987 \pm 127$ & $\mathrm{H}$ & 2457156.7194 & $148 \pm 37$ & $\mathrm{H}$ & 2455756.7940 & $-1980 \pm 45$ & $\mathrm{E}$ \\
2457152.6755 & $1959 \pm 217$ & $\mathrm{H}$ & 2457156.7306 & $452 \pm 38$ & $\mathrm{H}$ & 2455757.8761 & $-3673 \pm 47$ & $\mathrm{E}$ \\
2457152.7336 & $2114 \pm 64$ & $\mathrm{H}$ & 2457156.7671 & $1519 \pm 42$ & $\mathrm{H}$ & 2455960.1644 & $1991 \pm 47$ & $\mathrm{E}$ \\
2457152.7448 & $1867 \pm 50$ & $\mathrm{H}$ & 2457156.7783 & $1628 \pm 47$ & $\mathrm{H}$ & 2455961.0862 & $2281 \pm 46$ & $\mathrm{E}$ \\
2457152.8022 & $490 \pm 48$ & $\mathrm{H}$ & 2457156.7894 & $1870 \pm 49$ & $\mathrm{H}$ & 2455961.1488 & $1607 \pm 47$ & $\mathrm{E}$ \\
2457154.7847 & $-3701 \pm 34$ & $\mathrm{H}$ & 2457156.8006 & $1956 \pm 54$ & $\mathrm{H}$ & 2455966.1717 & $1859 \pm 84$ & $\mathrm{E}$ \\
2457154.8418 & $-3359 \pm 39$ & $\mathrm{H}$ & 2457156.8118 & $2054 \pm 63$ & $\mathrm{H}$ & 2455967.1499 & $1807 \pm 38$ & $\mathrm{E}$ \\
2457154.8530 & $-3152 \pm 38$ & $\mathrm{H}$ & 2457156.8230 & $2215 \pm 62$ & $\mathrm{H}$ & 2455971.1211 & $-3580 \pm 43$ & $\mathrm{E}$ \\
2457154.8642 & $-2976 \pm 42$ & $\mathrm{H}$ & 2457156.8608 & $2211 \pm 72$ & $\mathrm{H}$ & 2455971.1786 & $-2320 \pm 52$ & $\mathrm{E}$ \\
2457154.8754 & $-2682 \pm 45$ & $\mathrm{H}$ & 2457156.4955 & $-3953 \pm 43$ & $\mathrm{H}$ & 2456108.7415 & $-3726 \pm 39$ & $\mathrm{E}$ \\
2457154.8866 & $-2343 \pm 46$ & $\mathrm{H}$ & 2457156.8720 & $2137 \pm 97$ & $\mathrm{H}$ & 2456111.7426 & $2153 \pm 40$ & $\mathrm{E}$ \\
2457154.8978 & $-1986 \pm 48$ & $\mathrm{H}$ & 2457156.8832 & $2030 \pm 125$ & $\mathrm{H}$ & 2456115.7405 & $-3410 \pm 39$ & $\mathrm{E}$ \\
2457154.5078 & $1685 \pm 66$ & $\mathrm{H}$ & 2457156.8944 & $1908 \pm 219$ & $\mathrm{H}$ & 2456117.8275 & $1304 \pm 48$ & $\mathrm{E}$ \\
\hline
\end{tabular}

Notes. The columns give the Julian date, the mean longitudinal magnetic field measurement and the corresponding error bar. Measurements derived from HARPSpol (resp. ESPaDOnS) spectra are indicated with $\mathrm{H}$ (resp. E).

(Mikulášek et al. 2011), might be required to reconcile $\left\langle B_{z}\right\rangle$ and line profile variation.

\subsection{Cumulative Stokes V profiles}

The cumulative Stokes $V$ (CSV) profile diagnostic technique was suggested by Gayley \& Owocki (2014) and further developed by Kochukhov (2015). In this method the observed circular polarisation profiles of individual spectral lines or multi-line LSD profiles are converted to a spatially resolved measure of the longitudinal magnetic field density $\bar{B}_{\mathrm{z}}$, which essentially represents a Doppler-resolved equivalent of the widely used mean line-ofsight (longitudinal) magnetic field $\left\langle B_{z}\right\rangle$. In comparison to $\left\langle B_{z}\right\rangle$, which is a scalar quantity characterising the longitudinal magnetic field averaged over the entire stellar disk, $\bar{B}_{\mathrm{z}}$ is a velocitydependent measure of the line-of-sight field component averaged over the stripes of constant Doppler shift. Consequently, $\bar{B}_{\mathrm{z}}$ is less affected by cancellation of polarisation signals coming from regions with opposite field polarities and can provide a meaningful measure of the longitudinal field at crossover phases when $\left\langle B_{z}\right\rangle$ is zero. An analysis of CSV profiles allows one to qualitatively assess the topology of stellar magnetic field and to make an estimate of the local magnetic field strength without the need of sophisticated line profile modelling such as MDI. The CSV method is particularly suitable for fast-rotating stars suspected of hosting complex magnetic field geometries.

Here we have applied the CSV diagnostic procedure to the $\mathrm{Si}$ and Fe LSD Stokes $V$ profiles of HR 5624 and compared the resulting $\bar{B}_{\mathrm{z}}$ profiles to the predictions of the magnetic field geometry model derived by Bailey et al. (2012). According to this study, the field of HR 5624 is best approximated as a superposition of the aligned dipolar $B_{\mathrm{d}}=-9.6 \mathrm{kG}$, quadrupolar $B_{\mathrm{d}}=-23.2 \mathrm{kG}$ and octupolar $B_{\mathrm{oct}}=1.9 \mathrm{kG}$ components, along with $i=55^{\circ}$ and $\beta=78^{\circ}$.

The observed CSV profiles were obtained by a weighted average of the blue-to-red and red-to-blue integrals over the
Stokes $V$ profiles, normalised by the corresponding residual Stokes $I$ profiles (see Kochukhov 2015, for details). The resulting dynamic CSV spectra are presented in the panels a) and b) of Fig. 5. Each panel in this figure shows a phase variation (vertical axis) of the longitudinal field density (colour scale) spatially resolved in the direction (horizontal axis) perpendicular to the stellar rotational axis.

One can infer from Fig. 5 that the Si and Fe CSV profiles of HR 5624 are very similar despite a noticeable difference in the morphology of the underlying LSD Stokes $V$ profiles (modelled in Sect. 3.5). The CSV spectra of both chemical elements indicate a field topology with an extended region of weaker positive magnetic field (longitudinal field density $\bar{B}_{z} \leq 2.5 \mathrm{kG}$ ) and a smaller region of stronger negative field $\left(\bar{B}_{z} \geq-5.4 \mathrm{kG}\right)$. The CSV profiles are, therefore, generally compatible with a somewhat distorted and asymmetric dipolar field structure but do not reveal any evidence of a quadrupole-dominated field geometry.

The observed profiles can be compared with the theoretical CSV spectra obtained by integrating the line-of-sight component of the field geometry model by Bailey et al. (2012) for the same set of rotational phases as sampled by our spectropolarimetric observations. These theoretical CSV profiles, shown in Fig. 5c, are morphologically more complex than the observed ones. For example, the secondary negative magnetic-field region predicted by the model is not observed. Moreover, this field topology predicts a much stronger overall longitudinal magnetic field density $\left(\bar{B}_{z}\right.$ ranging from -11.3 to $8.5 \mathrm{kG}$ ) than is evident from the observed CSV data. Thus, prior to any MDI inversions, we are able to conclude, based on the CSV analysis results, that although the field geometry proposed by Bailey et al. (2012) successfully describes the observed phase variation of the disk-integrated longitudinal field, it is, in fact, incompatible with the observed Stokes $V$ profiles and appears to significantly overestimate both the strength and the degree of complexity of the surface magnetic field in HR 5624. These qualitative conclusions fully agree with an outcome of the MDI modelling presented below. 

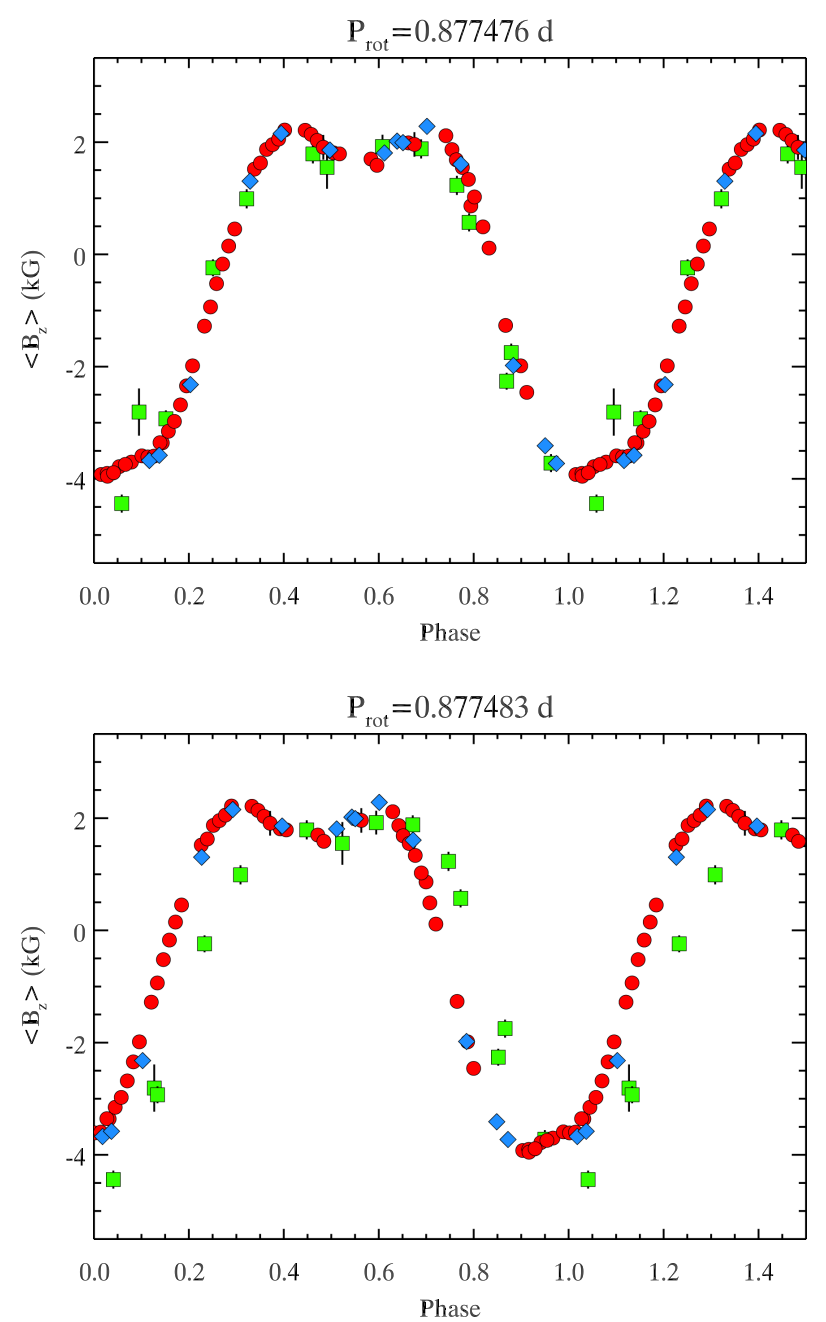

Fig. 4. Variation of the mean longitudinal magnetic field of HR 5624. The dark symbols show $\left\langle B_{z}\right\rangle$ measurements derived in this study from the HARPSpol (red circles) and ESPaDOnS (blue rhombs) observations. The green squares show the Balmer line photopolarimetric measurements by Landstreet (1990). The upper and lower panels illustrate phasing of the $\left\langle B_{z}\right\rangle$ data with the rotational periods $0.877476 \mathrm{~d}$ and $0.877483 \mathrm{~d}$, respectively.

\subsection{Magnetic Doppler imaging}

\subsubsection{Magnetic topology and distributions of $\mathrm{Si}, \mathrm{Cr}$, and $\mathrm{Fe}$}

We have reconstructed the magnetic field topology of HR 5624 by modelling the $\mathrm{Si}, \mathrm{Cr}$, and Fe LSD profiles with the help of the INVERSLSD MDI code described by Kochukhov et al. (2014). This inversion code performs simultaneous mapping of a vector magnetic field and one additional scalar parameter, in this case chemical abundance, based on an interpolation within pretabulated grids of theoretical local Stokes LSD profiles. Following the approach previously used for the magnetic mapping of CU Vir (Kochukhov et al. 2014), the local Stokes LSD profiles of HR 5624 were computed by applying the least-squares deconvolution procedure to a set of local synthetic four Stokes parameter spectra covering the entire observed wavelength range and including all relevant absorption lines (those comprising the $\mathrm{Si}, \mathrm{Cr}$, and Fe LSD masks as well as all significant blends). These theoretical spectra were tabulated for a grid of $15 \mathrm{limb}$ angles, 31 field strength values between 0 and $30 \mathrm{kG}, 15$ field vector orientations with respect to the line of sight, and 13-15 element abundance values. The element abundance variations
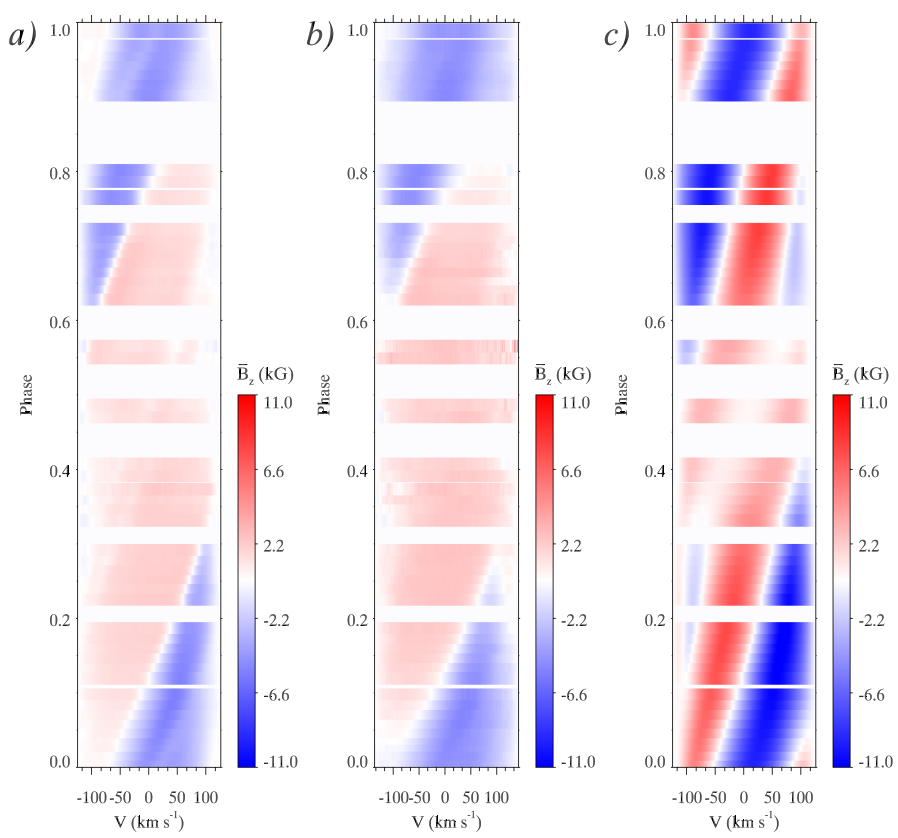

Fig. 5. Cumulative Stokes $V$ spectra corresponding to panel $a$ : the observed Fe LSD profiles; panel $b$ : the observed Si LSD profiles; and panel $c$ : the magnetic field geometry of HR 5624 reported by Bailey et al. (2012).

were treated with the help of three separate grids of LLMODELS atmospheres, calculated with the parameters determined above and changing logarithmic abundances of either $\mathrm{Si}, \mathrm{Cr}$, or $\mathrm{Fe}$ by 0.25 dex within the full range necessary for the respective inversions. This allowed us to take into account modifications of the local atmospheric structure and non-uniform continuum brightness (although according to Lehmann et al. 2007; Kochukhov et al. 2012; and Kochukhov 2017, these effects usually have only a marginal influence on the stellar surface maps).

The magnetic field geometry of HR 5624 was parameterised with a spherical harmonic expansion including modes with an angular degree up to $\ell_{\max }=20$. This value was chosen to approximately match the theoretical spatial resolution enabled by the $v_{\mathrm{e}} \sin i$ of HR 5624 (Fares et al. 2012). Both the poloidal and toroidal terms were included in the expansion. The inversions were initiated with zero magnetic field and a homogeneous abundance distribution. The regularisation parameters, controlling the strength of the harmonic regularisation for the magnetic field map and the Tikhonov regularisation for abundance maps, were adjusted as described by Kochukhov (2017). A series of MDI inversions was carried out for different values of the projected rotational velocity $v_{\mathrm{e}} \sin i$ and inclination angle $i$, aiming to find optimal values of these parameters. This analysis yielded $v_{\mathrm{e}} \sin i=106 \pm 2 \mathrm{~km} \mathrm{~s}^{-1}$ and $i=65 \pm 10^{\circ}$, both of which agree reasonably well with the determinations by Bailey et al. (2012).

The final fits by INVERSLSD to the observed Stokes $I$ and $V$ LSD profiles of $\mathrm{Si}, \mathrm{Cr}$, and Fe are presented in Figs. 6-8. The corresponding spherical plots of the total magnetic field strength, horizontal field, radial field and the field vector orientation are shown in Figs. 9-11. For comparison, we also illustrate in Fig. 12 the magnetic geometry model obtained in the previous detailed study of HR 5624 by Bailey et al. (2012). Finally, the harmonic energy distribution of the $\mathrm{Si}, \mathrm{Cr}$, and $\mathrm{Fe}$-based magnetic field maps is schematically illustrated in Fig. 14 for modes up to $\ell=5$ (the contribution of higher-order terms is negligible). Different statistical characteristics of these field geometries are summarised in Table 3. 


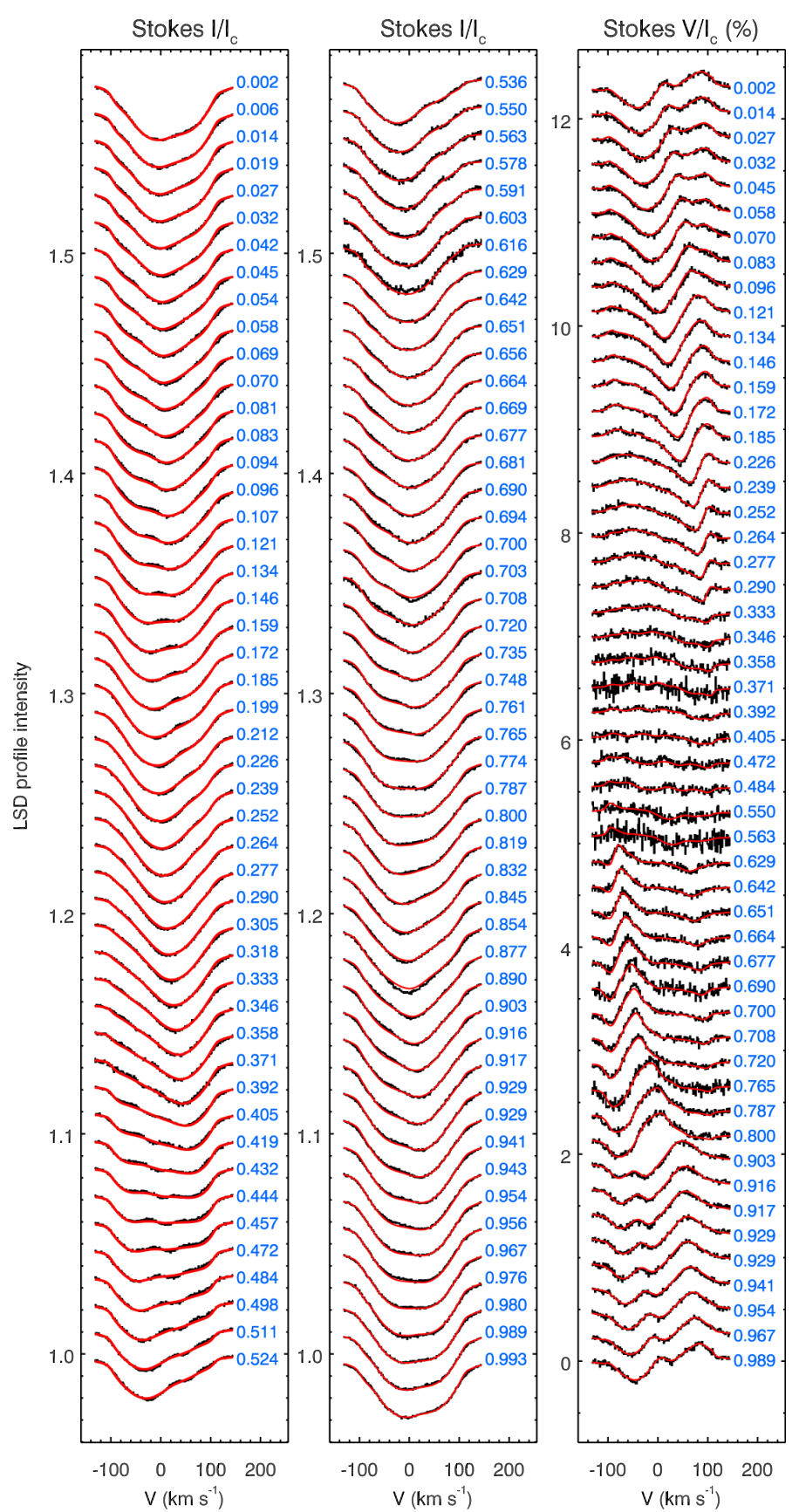

Fig. 6. Comparison of the observed Si LSD Stokes $I$ and $V$ profiles of HR 5624 with the fit achieved by the magnetic inversion code. Observations are shown with black histograms. Calculations for the final magnetic and chemical spot maps are shown with the solid red lines. Spectra corresponding to different rotation phases are offset vertically. Rotation phases are indicated to the right of each spectrum.

Turning attention to the magnetic field maps shown in Figs. 9-11, it is evident that the best-fitting magnetic field distribution is basically dipolar. However, this dipole is strongly asymmetric and distorted, with a much stronger and smaller negative magnetic pole where the radial field reaches $-11 \mathrm{kG}$ and a weaker, more extended positive magnetic field region with a radial field of up to $4.5-4.8 \mathrm{kG}$. The global mean of the field strength is found to be $4.0-4.4 \mathrm{kG}$. The maximum local field modulus is about $12 \mathrm{kG}$ in all three magnetic field maps. The corresponding mean field modulus is predicted to vary between 3.4 and $6.1 \mathrm{kG}$, with a sharp maximum at phase 0.95 and a broad
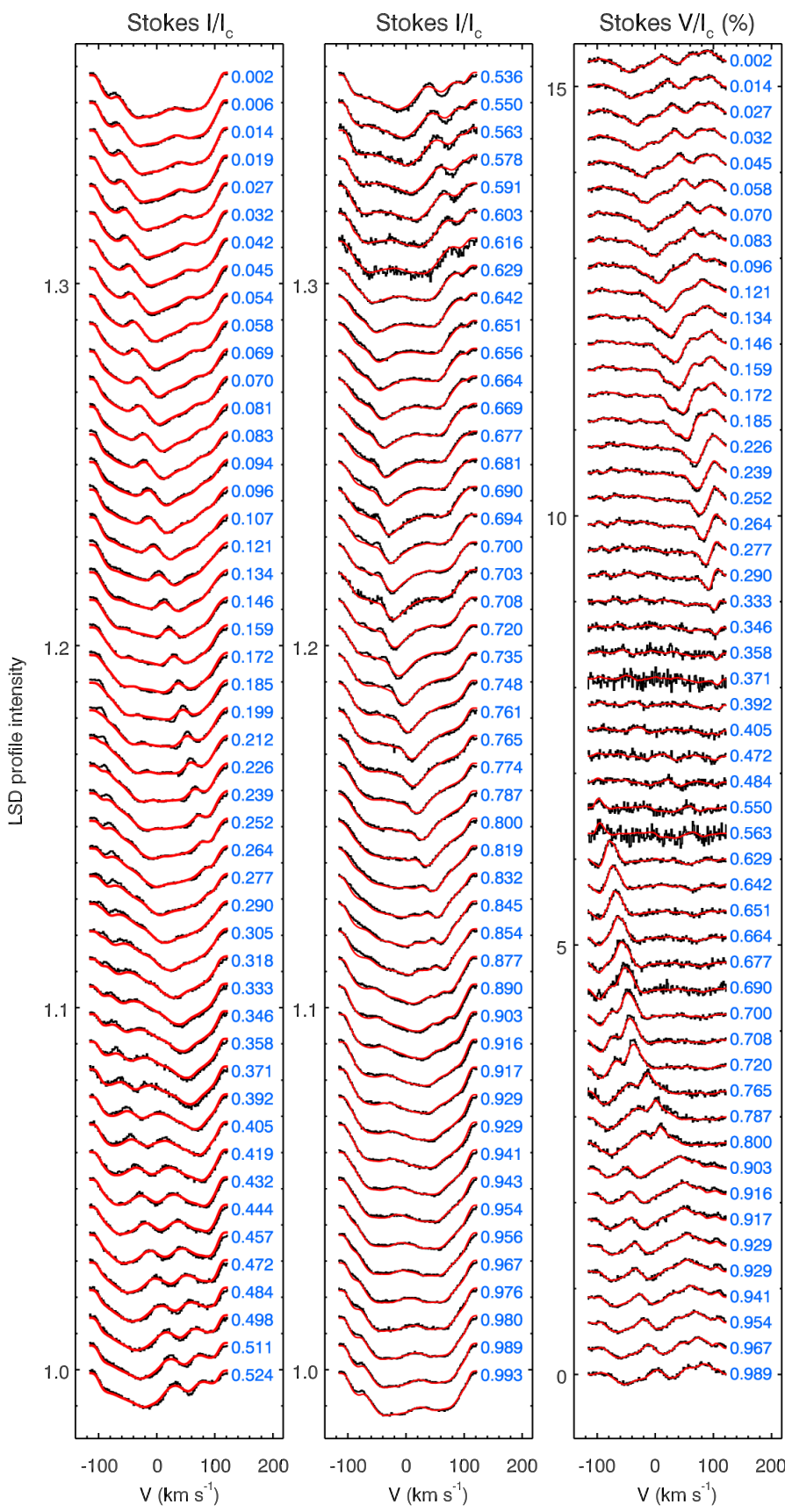

Fig. 7. Same as Fig. 6 for Cr LSD Stokes $I$ and $V$ profiles.

minimum in the phase interval $0.25-0.65$. The phase-averaged value of the field modulus is predicted to be $4.1-4.6 \mathrm{kG}$.

The details of the magnetic field topologies inferred from the independent LSD profile modelling of $\mathrm{Si}, \mathrm{Cr}$, and $\mathrm{Fe}$ agree very well, with typical local standard deviation of only $0.3-0.4 \mathrm{kG}$ for all three magnetic field vector components and the field modulus. This concordance is particularly noteworthy given the somewhat different appearance of the Fe and Cr Stokes $V$ LSD profiles (Figs. 7 and 8) and the significantly simpler morphology of the Si Stokes $V$ LSD profiles seen in Fig. 6. The latter appears to be a consequence of a less structured $\mathrm{Si}$ abundance distribution. All three magnetic field maps indicate that the negative pole is formed by two magnetic spots of unequal strength, with the trailing spot located at a longitude of about $45^{\circ}$ and including a substantial horizontal magnetic component. 


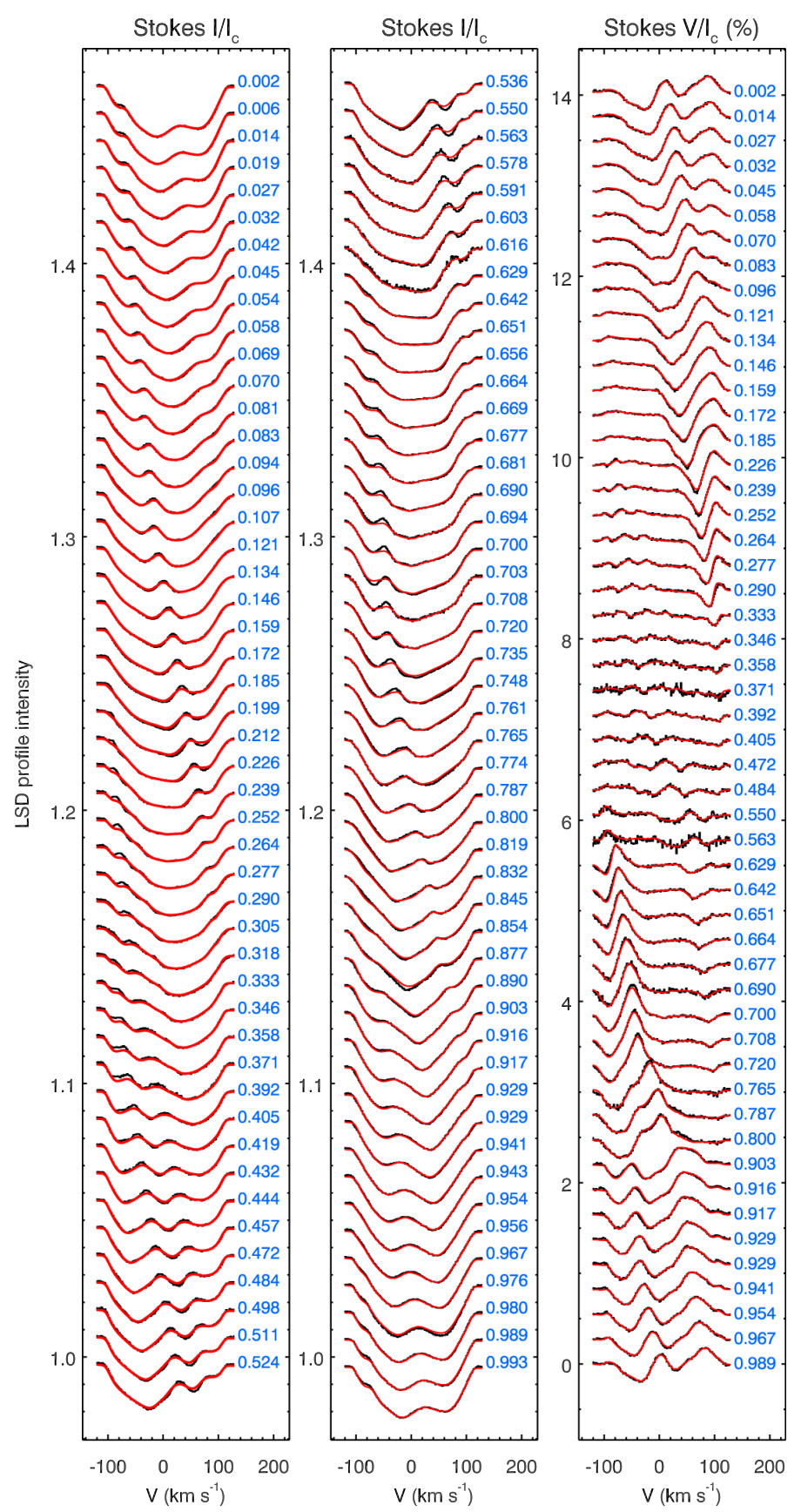

Fig. 8. Same as Fig. 6 for Fe LSD Stokes $I$ and $V$ profiles.

Comparing our MDI magnetic field maps with those of the quadrupole-dominated parametric magnetic field geometry $\left(B_{\mathrm{d}}=-9.6 \mathrm{kG}, B_{\mathrm{d}}=-23.2 \mathrm{kG} B_{\text {oct }}=1.9 \mathrm{kG}, i=55^{\circ}, \beta=78^{\circ}\right)$ proposed by Bailey et al. (2012), we find that our inversions favour much weaker global field strengths. In Fig. 13 we show forward calculations of $I$ and $V$ profiles based on the Bailey et al model. Although the $V$ profiles derived from the quadrupoledominated model show some qualitative resemblance to the observed profiles, they are systematically less structured and have amplitudes which are typically too large by factors of order 2 . On the other hand, although the $I$ line profiles resulting from the quadrupole-dominated model do not reproduce the detailed shape of the observations, they generally have about the right depth and show a reasonable resemblance to the observations. This better accord is probably due to the fact that the $I$ profiles are determined mainly by the adopted abundance distributions (particularly of $\mathrm{Fe}$ ) rather than the magnetic field.

The analysis of the harmonic energy distribution of the $\mathrm{Si}$, $\mathrm{Cr}$, and $\mathrm{Fe}$ magnetic maps (Fig. 14) reveals the dominant contribution (65-71\% in terms of the total magnetic field energy) of the dipolar component inclined by approximately $90^{\circ}$ with respect to the stellar rotational axis. All quadrupolar $(\ell=2)$ modes together contribute $20-26 \%$ of the magnetic energy. Contributions of higher order modes with $\ell \geq 3$ do not exceed $4.5 \%$. No evidence of a substantial toroidal field is found in our magnetic inversion results.

The $\mathrm{Si}, \mathrm{Cr}$, and $\mathrm{Fe}$ abundance distributions, reconstructed simultaneously with the magnetic field maps, are presented in Fig. 15. The chemical maps of these elements differ in detail but also have several properties in common. For instance, the contrast is about 2 dex in all three cases. Also, all three maps exhibit overabundance features coinciding with the stronger of the two magnetic spots at the negative pole as well as zones of relative element underabundance in the vicinity of the extended positive pole. For iron and chromium, this underabundance structure splits into two distinct narrow bands extended in latitude, possibly forming a ring centred at around longitude $190^{\circ}$. The effect of this underabundance ring is readily evident in the highly structured $\mathrm{Cr}$ and Fe Stokes I LSD profiles between phases 0.4 and 0.6. The $\mathrm{Cr}$ and $\mathrm{Fe}$ distributions are generally similar to each other. However, the chromium map shows several smaller overabundance spots at the rotational equator not present in the Fe map.

\subsubsection{Distributions of $\mathrm{Mg}, \mathrm{Ti}$, and $\mathrm{Nd}$}

In addition to the chemical abundance maps of $\mathrm{Si}, \mathrm{Cr}$, and $\mathrm{Fe}$ recovered from the Stokes $I$ and $V$ LSD profiles as part of the MDI inversions, we also studied the surface distributions of several other elements using intensity profiles of individual spectral lines. Due to the rapid rotation of HR 5624, it is difficult to find unblended lines of elements other than $\mathrm{Si}, \mathrm{Cr}$, and Fe. Nevertheless, surface distributions of $\mathrm{Mg}, \mathrm{Ti}$, and $\mathrm{Nd}$ could be derived by modelling the 448.1, 480.5, and $494.3 \mathrm{~nm}$ lines of these elements, respectively. Chemical abundance maps were obtained using INVERS10 (Piskunov \& Kochukhov 2002; Kochukhov \& Piskunov 2002) and adopting the average of the magnetic field topologies determined above. In each case the line list included up to ten blending lines in addition to the main spectral features. Most of the secondary lines belonged to $\mathrm{Fe}$ and $\mathrm{Cr}$, allowing the treatment of inhomogeneous distributions of these elements according to the previously derived abundance maps.

The resulting surface distributions of $\mathrm{Mg}, \mathrm{Ti}$, and $\mathrm{Nd}$ are presented in Fig. 16. All three elements exhibit horizontal inhomogeneities with the abundance contrast of 1.6-2.8 dex. Some of these surface distributions show certain similarities to the $\mathrm{Si}, \mathrm{Cr}$, and $\mathrm{Fe}$ abundance maps recovered from the LSD profiles. The distribution of $\mathrm{Nd}$ is dominated by two distinct overabundance areas, seen at the rotational phases 0.2 and 0.8 , which are offset from the magnetic equator by $\sim 30^{\circ}$ in longitude towards the positive magnetic field regions. Curiously, the Si map is also dominated by two spots, with one of them almost coinciding with the spot of $\mathrm{Nd}$ (phase 0.2 ), but another one offset by nearly $60^{\circ}$ from the second $\mathrm{Nd}$ spot (phase 0.8 ). The region with the minimum element abundance in the vicinity of the extended, weak-field, positive magnetic pole occurs, in one form or another, in every abundance map. On the other hand, with the exception of Nd, no such region is seen close to the strong-field, negative magnetic pole. 

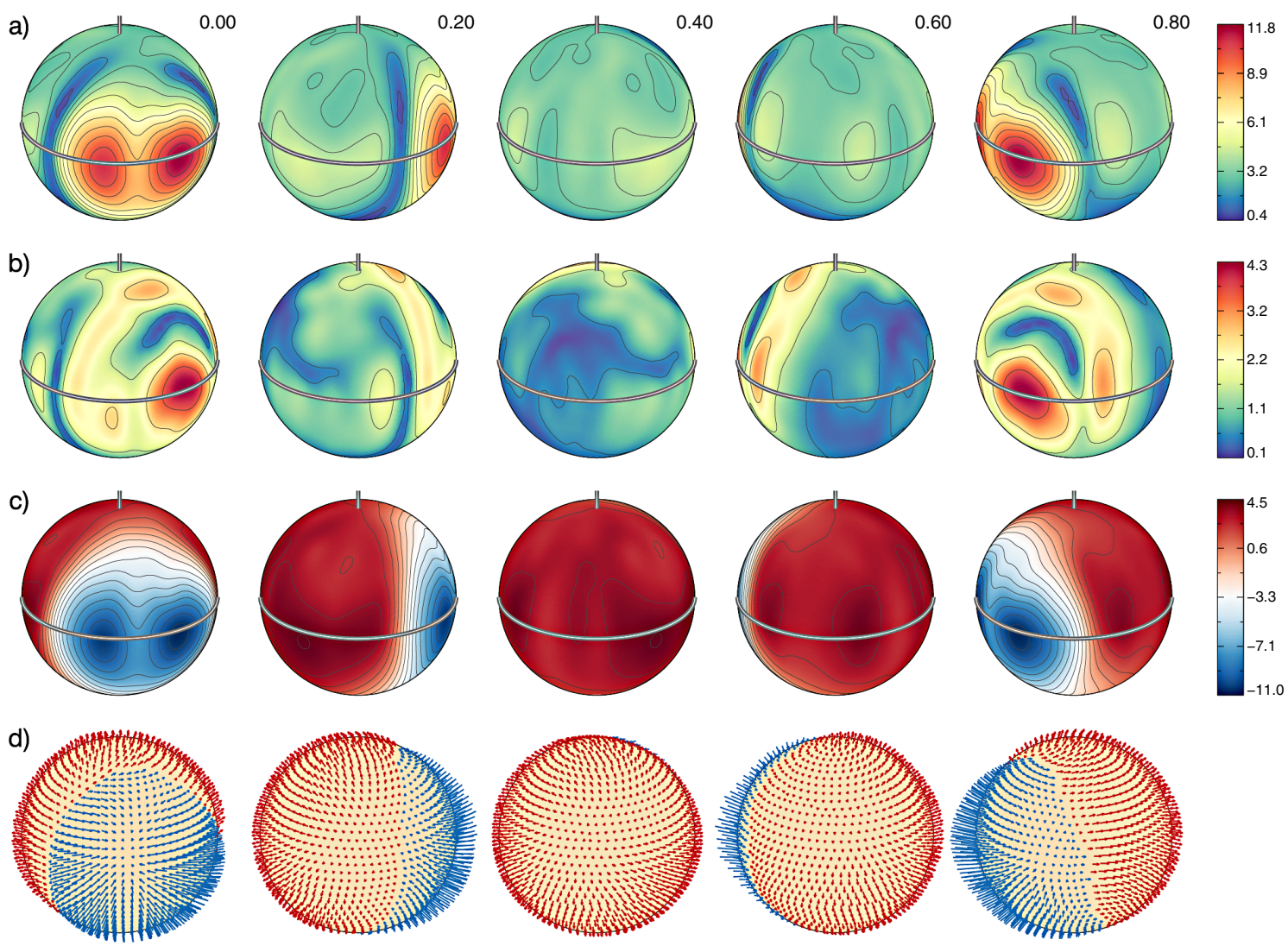

Fig. 9. Surface magnetic field distribution of HR 5624 derived from the Si LSD profiles. The star is shown at five rotation phases, which are indicated above each spherical plot column. The inclination angle is $i=65^{\circ}$. The spherical plots show the maps of panel $a$ : field modulus; panel $b$ : horizontal field; panel $c$ : radial field; and panel $d$ : field orientation. The contours over spherical maps are plotted with a step of $1 \mathrm{kG}$. The thick line and the vertical bar indicate positions of the rotational equator and the pole, respectively. The colour-bars give the field strength in kG. The two different colours in the field orientation map correspond to the field vectors directed outwards (red) and inwards (blue).

Table 3. Statistical characteristics of the magnetic field maps reconstructed from Si, Cr, and Fe LSD profiles of HR 5624.

\begin{tabular}{|c|c|c|c|c|c|c|c|}
\hline \multirow[t]{2}{*}{ Element } & \multicolumn{2}{|c|}{ Local field strength $(\mathrm{kG})$} & \multicolumn{2}{|c|}{ Mean field modulus (kG) } & \multirow{2}{*}{$\begin{array}{c}E_{\ell=1} / E_{\text {tot }} \\
(\%)\end{array}$} & \multirow{2}{*}{$\begin{array}{c}E_{\ell=2} / E_{\mathrm{tot}} \\
(\%)\end{array}$} & \multirow{2}{*}{$\begin{array}{c}E_{\mathrm{pol}} / E_{\mathrm{tot}} \\
(\%)\end{array}$} \\
\hline & Mean & Range & Mean & Range & & & \\
\hline $\mathrm{Si}$ & 3.95 & $0.44-11.72$ & 4.06 & $3.19-5.69$ & 70.0 & 20.1 & 95.3 \\
\hline $\mathrm{Cr}$ & 4.36 & $0.28-11.95$ & 4.57 & 3.56 & 70.6 & 20.9 & 93.8 \\
\hline $\mathrm{Fe}$ & 4.27 & $0.45-12.14$ & 4.38 & $3.34-6.20$ & 65.4 & 25.6 & 94.4 \\
\hline
\end{tabular}

\section{Discussion}

\subsection{Magnetic field topology of HR5624}

In this study we have carried out the first detailed polarisation line profile modelling of the young Bp star HR 5624, a star previously suggested to host an unusual, predominantly quadrupolar, very strong surface magnetic field (Landstreet 1990; Bailey et al. 2012). Contrary to these predictions, our MDI inversions reveal a much weaker magnetic field, which from a first approximation resembles a distorted, offset dipole. This field topology still resembles a nearly aligned superposition of a dipole and a linear quadrupole. Moreover, our finding that the negative magnetic pole is comprised of a pair of high-contrast magnetic features qualitatively agrees with the conjecture of previous quadrupolar models, which predicted a strong magnetic spot at the same location. These results appear to reinforce the notion that an aligned dipole plus quadrupole magnetic field parameterisation provides a satisfactory first-order description of Ap-star magnetic fields and is useful for coarse analyses of large stellar samples (e.g. Landstreet \& Mathys 2000).
However, despite this qualitative agreement with our detailed MDI maps, other predictions of the parametric field topology models are entirely spurious. In the specific case of HR 5624, quantitative MDI results differ markedly from the corresponding quadrupolar model predictions. MDI maps indicate that the dipolar component dominates the stellar field topology, contributing $65-70 \%$ of the total magnetic field energy, while all quadrupolar $(\ell=2)$ modes together provide a factor of 2.6-3.5 less contribution to the magnetic energy budget. This is a much larger dipolar contribution compared to the findings of previous field topology studies by Landstreet (1990) and Bailey et al. (2012), both of which adopted a combination of aligned dipole, linear quadrupole, and linear octupole. The first paper found a fairly wide variety of parameter combinations that could explain the observed $\left\langle B_{z}\right\rangle$ variations, with models based on a realistic limbdarkening function and (arbitrarily assumed) $i=90^{\circ}$ requiring a quadrupole-to-dipole polar field strength ratio of $B_{\mathrm{q}} / B_{\mathrm{d}} \sim 3$. The more recent study by Bailey et al. (2012) inferred $B_{\mathrm{q}} / B_{\mathrm{d}}=2.4$ for a stellar inclination similar to the one found in our study. These high $B_{\mathrm{q}} / B_{\mathrm{d}}$ values correspond to $>80 \%$ contribution of 
O. Kochukhov et al.: Magnetic field topology and chemical spots of HR 5624
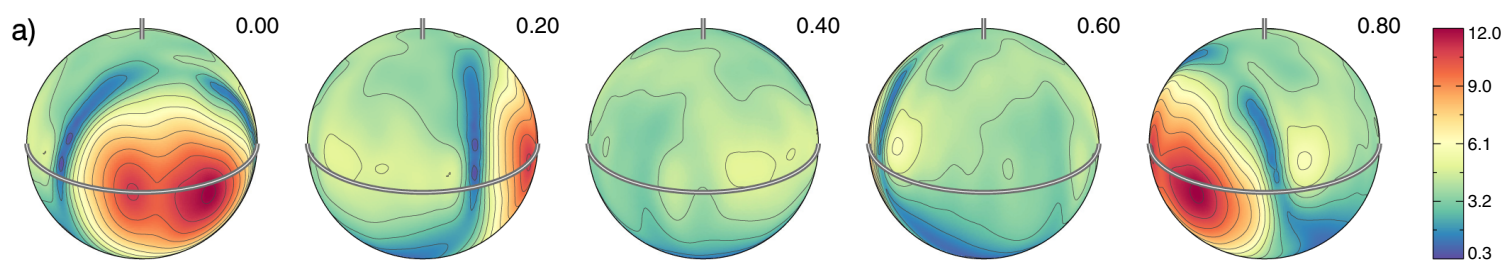

b)
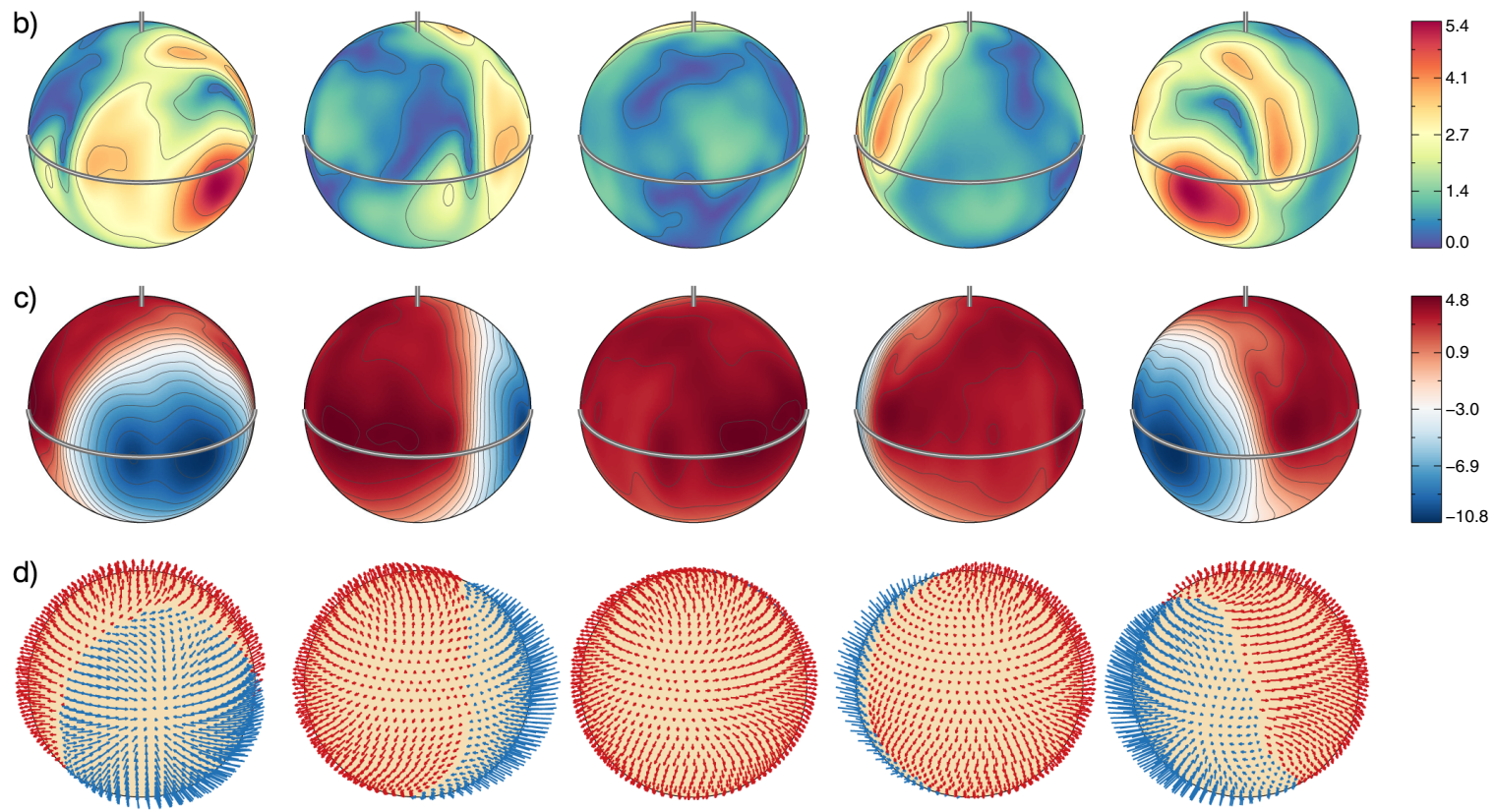

Fig. 10. Same as Fig. 9 for the surface magnetic field distribution derived from the Cr LSD profiles.
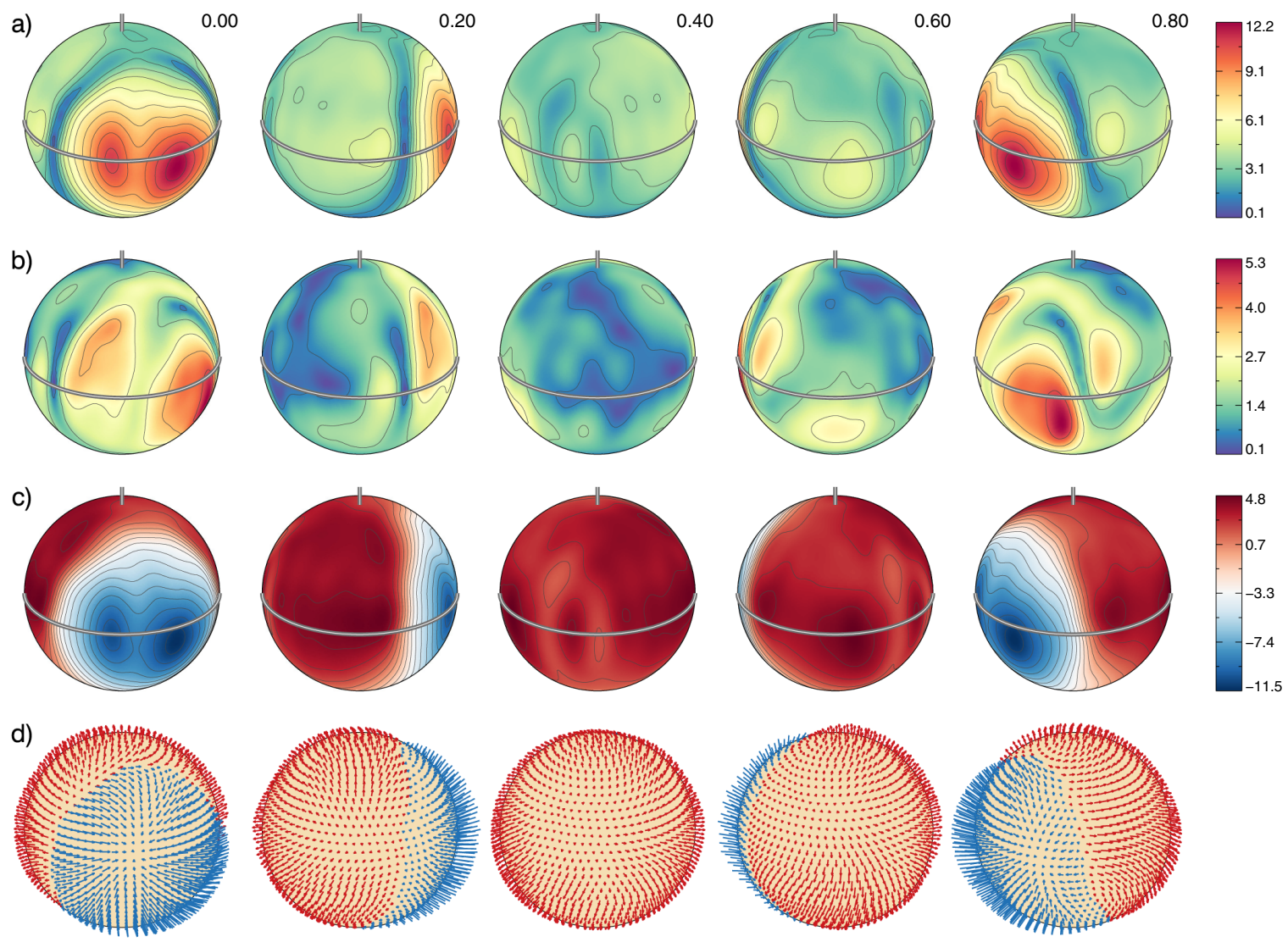

Fig. 11. Same as Fig. 9 for the surface magnetic field distribution derived from the Fe LSD profiles. 

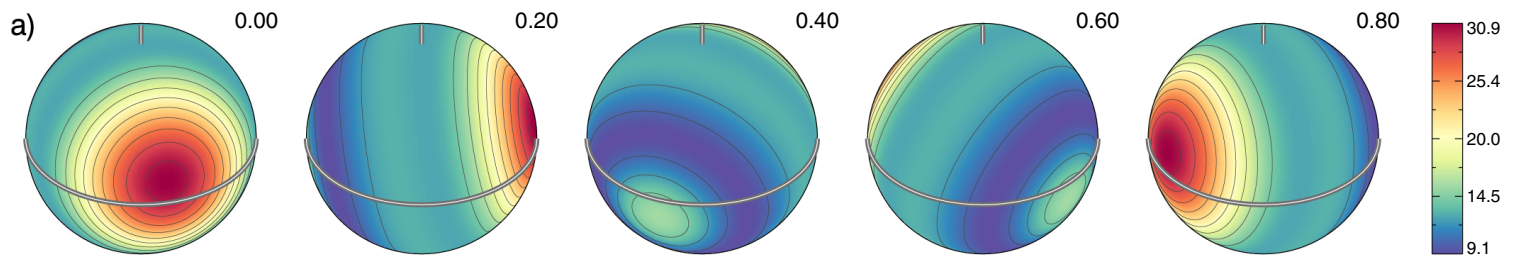

b)
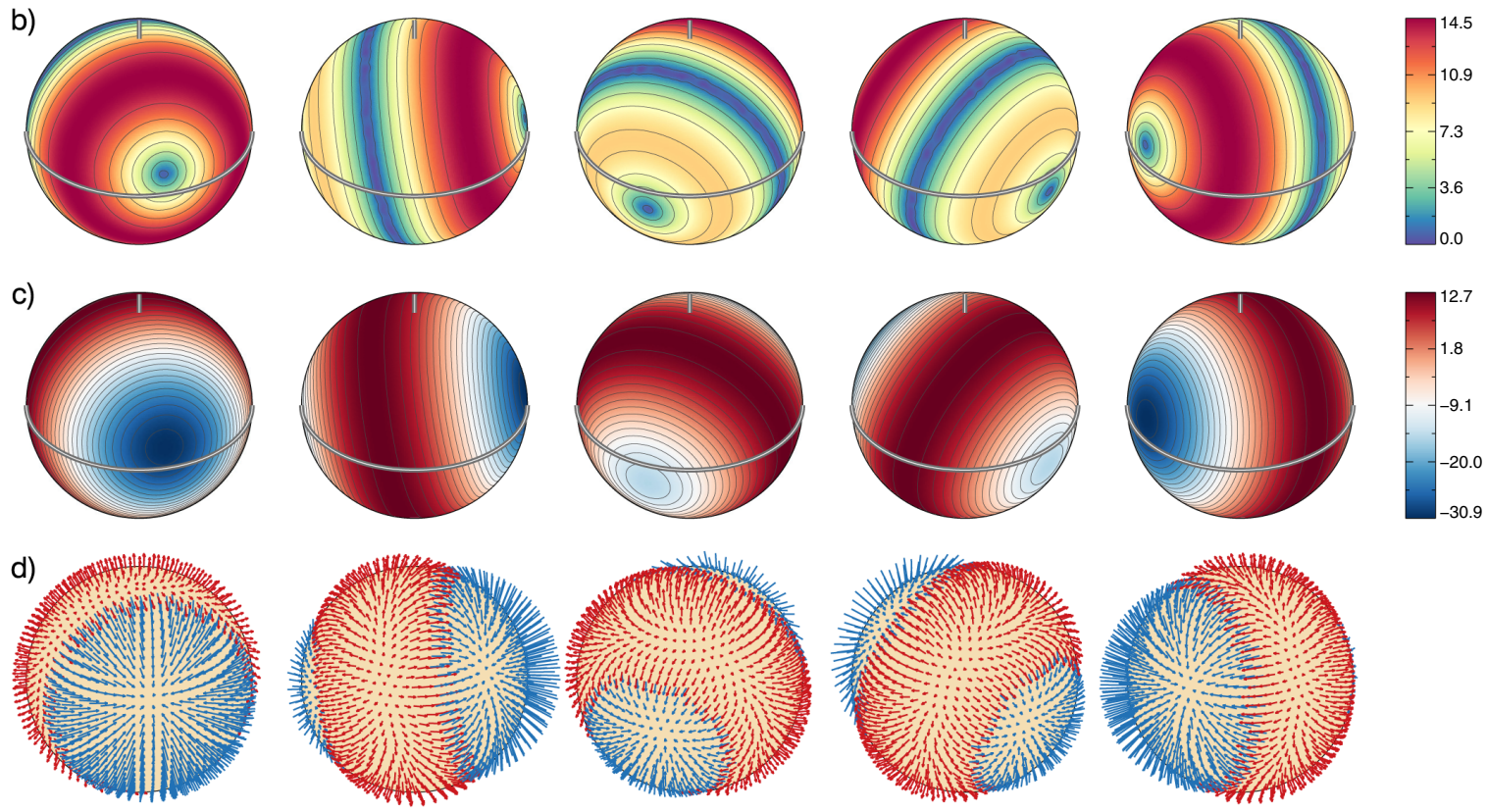

Fig. 12. Same as Fig. 11 for the surface magnetic field distribution derived by Bailey et al. (2012). The contours over spherical maps are plotted with a $2 \mathrm{kG}$ step.

$\ell=2$ harmonic modes to the total magnetic energy. In addition, both our mean and maximum local magnetic field strengths are lower than the values suggested by Landstreet (1990) and Bailey et al. (2012) by about a factor of 2-3.

Unlike the previous magnetic field studies of HR 5624, which were limited to fitting longitudinal magnetic field measurements and magnetic line broadening, we considered the full Stokes $I$ and $V$ line profile information, took into account surface chemical inhomogeneities, and derived consistent results from independent modelling of three chemical elements. The resulting best-fitting MDI magnetic field maps successfully explain both the polarisation profiles themselves and their low-order moments, including the mean longitudinal magnetic field. Thus, our magnetic field geometry model passes a more rigorous observational test and is therefore more reliable. On the other hand, as shown above, the magnetic geometry proposed by Bailey et al. (2012) fails to reproduce the amplitude and detailed shapes of the Stokes $V$ profiles.

The magnetic field analysis by Bailey et al. (2012) included an attempt to estimate the mean magnetic field modulus from the differential Zeeman broadening of two pairs of spectral lines with different effective Landé factors. This methodology, expected to be reliable for $v_{\mathrm{e}} \sin i$ of up to $50 \mathrm{~km} \mathrm{~s}^{-1}$ (Bailey 2014), yielded mean field strength in the range of 11-23 kG for HR 5624, which is incompatible with our results. Examining synthetic and observed spectra of HR 5624 in the vicinity of the four Cr II and Fe II lines used by Bailey et al. (2012), we find that the profiles of all these absorption features are strongly blended by other lines and are significantly distorted by chemical abundance inhomogeneities. This makes it impossible to reliably isolate the $\mathrm{Cr}$ II and Fe II lines in question, let alone measure their widths with a precision of better than $0.5 \%$ as implied by $2.5 \mathrm{kG}$ field strength errors quoted by Bailey et al. (2012). Hence, it is likely that their mean field modulus measurements are entirely spurious.

Compared to the previous restricted parametric low-order multipolar models used by Landstreet (1990) and Bailey et al. (2012), our MDI code allows many more degrees of freedom corresponding to all possible poloidal and toroidal modes in a general spherical harmonic expansion. We also impose an additional regularising constraint forcing the global magnetic energy to a minimum, which dampens contributions of higher-order modes. Perhaps, in hindsight, it is not surprising that a model with a larger number of free parameters yields a weaker overall magnetic field with a lower quadrupolar contribution. Nevertheless, the fact that this model fits the observed polarisation profiles so successfully indicates that the quadrupole-dominated field structure previously proposed for HR 5624 was merely a consequence of adopting a specific, highly restrictive low-order multipolar field parameterisation rather being linked to particular characteristics of the observational data. In this respect, our MDI analysis of HR 5624 follows a series of polarisation profile studies of $\mathrm{Ap} / \mathrm{Bp}$ stars, which demonstrate frequent failures of the classical multipolar field models based on fitting the longitudinal field curves and other observables derived from Stokes profiles to match the line profile themselves (Bagnulo et al. 2001; Kochukhov et al. 2004, 2011). It appears that predictions of such models are only fairly robust when dealing with simple, dipoledominated field geometries and become increasingly misleading whenever they attempt to incorporate large deviations from a dipolar field structure.

We may also compare the results of our detailed mapping with the chemical abundances derived by Bailey et al. (2012). Because of the largely different modelling assumptions, detailed 


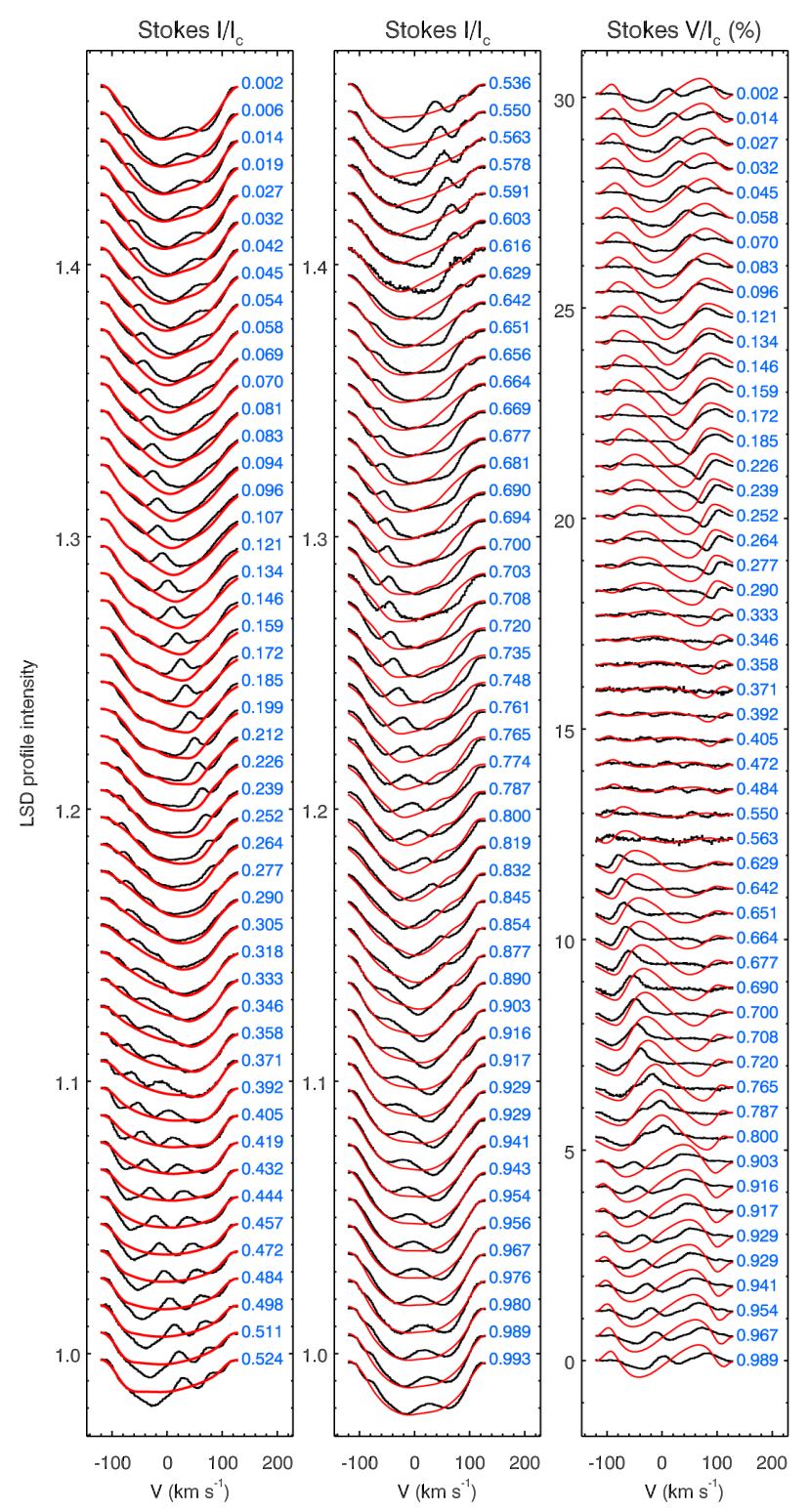

Fig. 13. Same as Fig. 6 for the comparison of the observed Fe LSD Stokes $I$ and $V$ profiles and forward calculations with the iron abundance distribution and magnetic field geometry of Bailey et al. (2012).

comparisons are difficult to achieve. We note that in the case of the elements $\mathrm{Mg}, \mathrm{Si}, \mathrm{Cr}$, and $\mathrm{Fe}$, however, the abundances derived by Bailey et al. lie within the range of abundances in our maps, although generally with a smaller range of variation (consistent with the very low spatial resolution of Bailey et al. surface mapping). In the cases of $\mathrm{Ti}$ and $\mathrm{Nd}$, the abundances derived by Bailey et al. are near the low-abundance end of the range found here. Thus, in general, chemical abundances derived from the simple model seem to be fairly indicative of our much more accurate values, and in any case do not lead to spuriously large overabundances.

To summarise, the main outcome of our MDI analysis of HR 5624 is that the dominant contribution to its field topology is provided by a distorted dipole rather than an axisymmetric (linear) quadrupole. Polarisation profile modelling of HD 37776 , also believed to have a very strong quadrupolar field, yielded similar results, although in that case the best-fitting magnetic map was comprised of smaller-scale magnetic spots without an underlying dipole-like structure (Kochukhov et al. 2011).
Considering that HR 5624 and HD 37776 were the best candidates for a quadrupole-dominated field topology known among $\mathrm{Ap} / \mathrm{Bp}$ stars, there seems to be no remaining evidence that such hypothetical magnetic field geometries occur in real stars. Interestingly, this conclusion is in line with the results of three-dimensional numerical simulations of the fossil magnetic field evolution in stably stratified stellar interiors. The studies by Braithwaite \& Nordlund (2006) and Braithwaite (2008) have demonstrated that initially random magnetic field quickly develops into either a dipole-like topology or a complex, non-axisymmetric configuration, depending on the initial radial distribution of the field energy. The surface magnetic field structures of the dipole-like models calculated by Braithwaite (priv. commun.) can, in fact, be quite distorted, showing various dipole offsets, toroidal contributions, and smaller-scale magnetic features not unlike the pair of spots at the negative magnetic pole of HR 5624 or those found in the Stokes IQUV inversions of $\alpha^{2}$ CVn (Kochukhov \& Wade 2010; Silvester et al. 2014). On the other hand, none of these theoretical magnetohydrodynamic models yield a surface field configuration reminiscent of a linear quadrupole.

\subsection{Comparison with diffusion theory predictions}

Atomic diffusion in the presence of a magnetic field is believed to be the main mechanism responsible for non-solar chemical abundance patterns and chemical inhomogeneities in the surface layers of Ap/Bp stars (Michaud et al. 2015). Equilibrium diffusion models, such as those calculated by LeBlanc et al. (2009) and Alecian \& Stift (2010), predict significant vertical element stratification in the atmospheric line-forming region. These vertical stratification profiles presumably change across the stellar surface according to the local magnetic field geometry, giving rise to the rotational line profile modulation interpreted by DI codes as chemical spots. As summarised by Ryabchikova (2008) and Ryabchikova et al. (2011), stratification profiles predicted by diffusion calculations agree qualitatively and, in some cases, quantitatively with the findings of observational studies, especially those targeting cool Ap stars, which exhibit the most pronounced vertical abundance gradients (e.g. Kochukhov et al. 2006; Ryabchikova et al. 2008; Shulyak et al. 2009; Nesvacil et al. 2013).

On the other hand, no satisfactory agreement can be found between the observed DI maps and theoretically predicted horizontal abundance structures. It appears that the current equilibrium diffusion calculations predict no variation of chemical stratification over the stellar surface except in narrow bands coinciding with horizontal field regions. For example, according to Alecian \& Stift (2010), the iron vertical stratification profiles calculated for $T_{\text {eff }}=12000 \mathrm{~K}, \log g=4.0$ atmospheric models are independent of the field orientation until the field vector is inclined by more than $75-80^{\circ}$ with respect to the local surface normal. Almost all other chemical elements, including Si and $\mathrm{Mg}$ studied here, are predicted to follow the exact same pattern, which would be interpreted by Doppler inversions as narrow overabundance rings located at the magnetic equator. More sophisticated time-dependent diffusion calculations (Alecian et al. 2011; Stift \& Alecian 2016) have yet to be published in a format enabling a direct comparison with MDI maps. However, despite some changes in the vertical element distributions, there are no indications that these time-dependent calculations would lead to any other horizontal pattern besides narrow overabundance rings. 

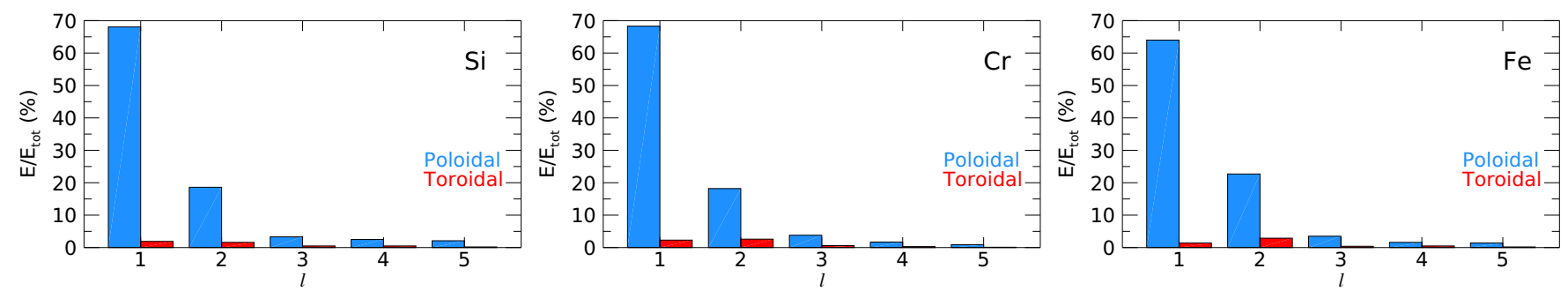

Fig. 14. Relative energies of the poloidal and toroidal harmonic modes with different angular degrees $\ell$ for the magnetic field topology of HR 5624 derived from $\mathrm{Si}$ (left), $\mathrm{Cr}$ (middle), and Fe (right) LSD profiles.
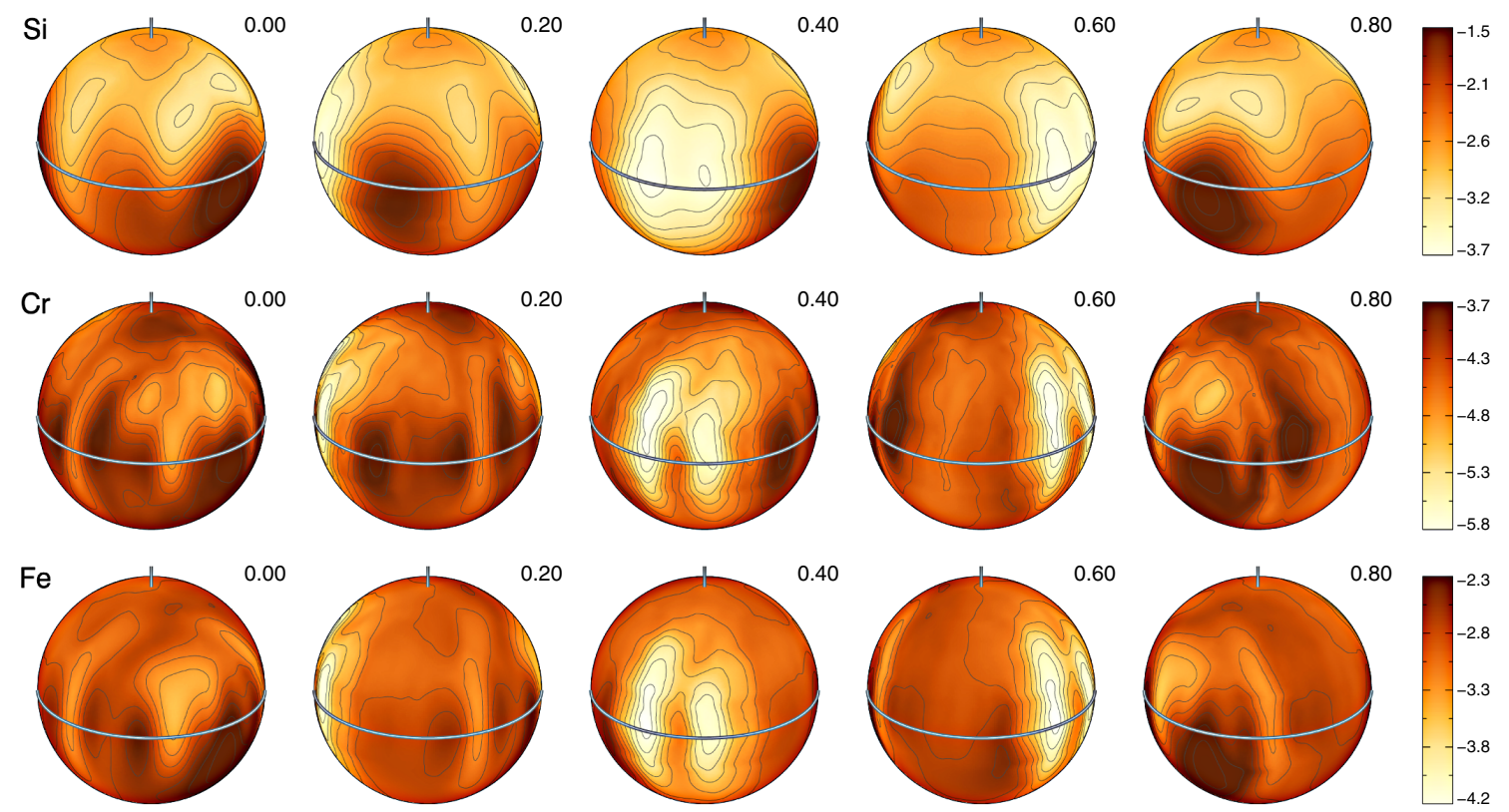

Fig. 15. Silicon (top), chromium (middle), and iron (bottom) surface abundance distributions reconstructed from the LSD profiles of these elements simultaneously with the magnetic field mapping. The star is shown at five rotational phases (five columns), as indicated next to each plot. The contours over spherical maps are plotted with a 0.2 dex step. The side bars give element abundances in logarithmic units $\log N_{\mathrm{el}} / N_{\text {tot }}$.

Taking advantage of the detailed information about the magnetic field geometry of HR 5624, and several chemical abundance maps reconstructed self-consistently with the magnetic field, we attempted to carry out a quantitative comparison of our observational data with the theoretical element distribution models initially presented by Alecian \& Stift (2010) and updated by Alecian (2015). It should be emphasised that HR 5624 is a particularly suitable target for such a comparison due to its high projected rotational velocity. For stars with low $v_{\mathrm{e}} \sin i$, one can plausibly argue that lateral variation of the vertical stratification profile might lead to changes of the local line shapes that interfere with the usual DI inversion based on the assumption of a single value of abundance for each surface point. However, for stars with $v_{\mathrm{e}} \sin i$ as high as $\sim 100 \mathrm{~km} \mathrm{~s}^{-1}$, details of the local line profiles are irrelevant (e.g. Unruh \& Collier Cameron 1995), meaning that the standard DI inversion should provide a reliable estimate of the mean local element abundance.

Given the high quality of the Fe and Si LSD Stokes I profiles obtained for HR 5624, we decided to assess diffusion theory predictions for these two elements. However, despite the presence of bi-dimensional Si maps in the publication by Alecian \& Stift (2010), no meaningful predictions can be made for this element according to Alecian (2015) because $\mathrm{Si}$ is unsupported by the radiative pressure everywhere on the stellar surface. This results in a sub-solar abundance of this element, contrary to the wellestablished large overabundance of Si in HR 5624 and many other Si-rich Bp stars. Limiting our analysis to Fe alone, we calculated the local field orientation according to our Fe MDI results. The corresponding absolute field inclination map shown in Fig. 17 agrees very well with the field inclination distributions inferred from the Si and Cr LSD profile inversions. This map shows a slightly warped ring of high field inclination wrapped around the star. The inferred location of the horizontal field zones is very robust since it is mostly determined by the radial field component, which is well-constrained for a dipole-like field even in the absence of the Stokes $Q$ and $U$ data.

Considering the $\mathrm{Fe}$ field inclination map, we adopted $\log N_{\mathrm{Fe}} / N_{\mathrm{tot}}=-2.0$ for the regions where the field inclination exceeded $75^{\circ}$ and $\log N_{\mathrm{Fe}} / N_{\mathrm{tot}}=-3.5$ elsewhere. These abundances were adopted to approximately represent the inferred maximum and mean Fe concentration, respectively. The exact abundance values are less important for the following qualitative discussion of line profile variability compared to the position and size of element overabundance regions.

Unsurprisingly, given the tiny surface fraction (about 3\%) occupied by field of high inclination and therefore high $\mathrm{Fe}$ abundance, this element distribution model yields a very weak, smallscale Stokes $I$ line profile variation (red solid line in Fig. 18), which is entirely unlike the broad, high-amplitude distortions travelling across the observed Fe line profiles. Trying to increase the area of $\mathrm{Fe}$ overabundance regions, we generated another map with $\log N_{\mathrm{Fe}} / N_{\mathrm{tot}}=-2.0$ adopted for the zones with 

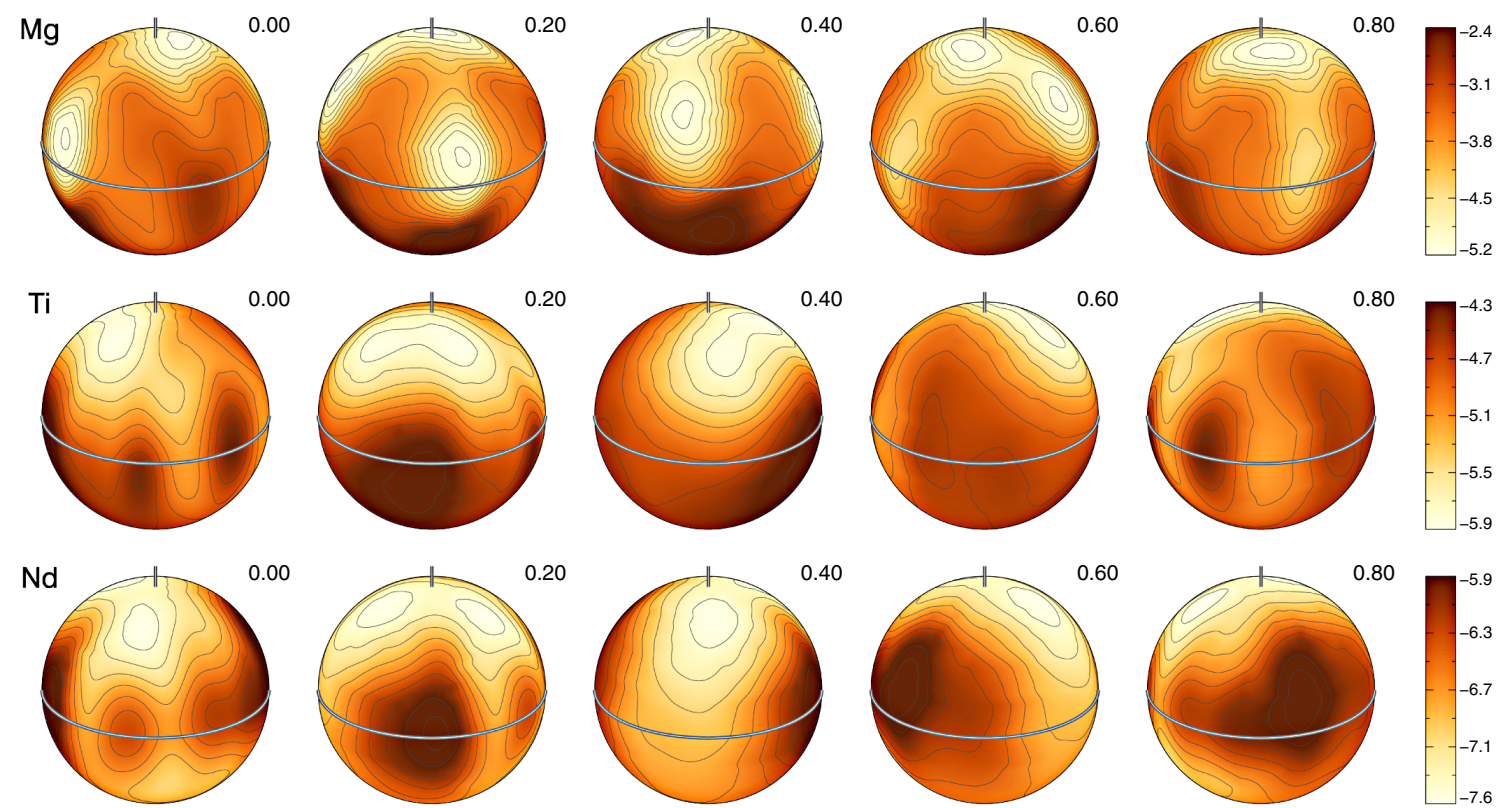

Fig. 16. Same as Fig. 15 for magnesium, titanium, and neodymium surface abundance distributions reconstructed from individual spectral lines of these elements.
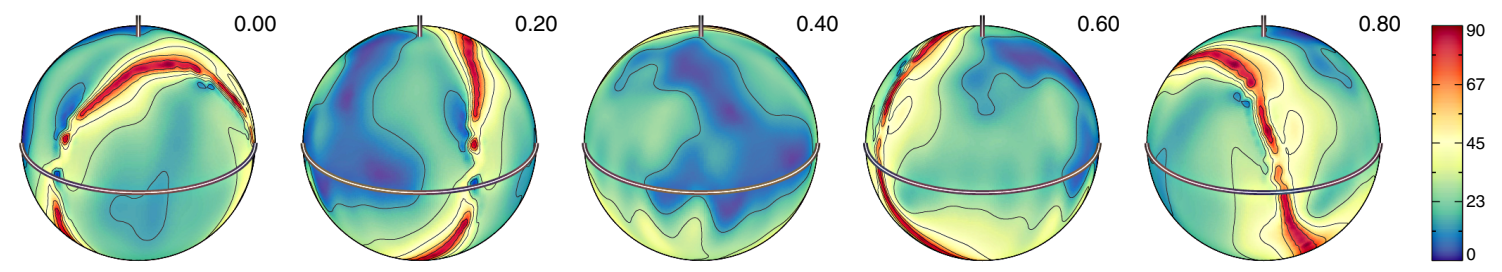

Fig. 17. Angle between the local field vector and the surface normal corresponding to the magnetic field maps reconstructed from the Fe LSD profiles (see Fig. 11). The contours over spherical maps are plotted with a step of $15^{\circ}$.

field inclination exceeding $45^{\circ}$. The resulting profile variation, shown by the dashed line in Fig. 18, is more discernible but is still vastly different compared to observations. In fact, there is an anti-correlation between dips in the model profiles and bumps in observations, indicating that $\mathrm{Fe}$ is underabundant instead of overabundant in the regions characterised by a high field inclination.

According to the diffusion calculations at $T_{\text {eff }}=10000 \mathrm{~K}$ (Alecian 2015), the $\mathrm{Cr}$ abundance distribution is expected to be very similar to the one for $\mathrm{Fe}$, that is, dominated by a narrow overabundance ring coinciding with the magnetic equator. Given the similarity of the $\mathrm{Cr}$ and Fe line profile variation in HR 5624, this hypothetical chromium distribution would evidently lead to the same disagreement with observations as discussed above for Fe. On the other hand, earlier calculations by Alecian \& Stift (2010) seem to indicate almost no horizontal $\mathrm{Cr}$ abundance variation at $T_{\text {eff }}=12000 \mathrm{~K}$, in conflict with the high-contrast $\mathrm{Cr}$ distribution map inferred in our study and with conspicuous $\mathrm{Cr}$ line variability often observed in Bp stars in this temperature range.

It is beyond the scope of this paper to address the reasons for this apparent failure of the diffusion theory to match the observed surface abundance structures. Several physical processes that can potentially alter predictions of the current diffusion models have been enumerated by Alecian (2015). Among various effects currently overlooked by these calculations, the anisotropic mass loss already known to be important in the context of atmospheric diffusion (Babel 1992) is particularly relevant. Perhaps, a more successful diffusion model can be developed by imposing the outer atmospheric boundary condition according to the magnetically confined (Babel \& Montmerle 1997; Townsend et al. 2005), chemically fractionated (Krtička 2014) stellar wind treatment. In addition, the current-driven diffusion effects (Urpin 2016) and the global hydrodynamical instabilities likely responsible for the low-contrast dynamic spots on $\mathrm{HgMn}$ stars (Kochukhov et al. 2007; Korhonen et al. 2013) should be seriously looked into. In any case, our assessment shows that the current theoretical diffusion modelling of the horizontal chemical abundance structures is far too simplistic and needs to be significantly improved before the output of these calculations can be meaningfully compared to the empirical DI maps of Ap/Bp stars.

\section{Conclusions}

In this paper we present the first DI and MDI analysis of HR 5624. This is a young, rapidly rotating, magnetic B-type chemically peculiar star with a well-known age due to its cluster membership. HR 5624 is famous for its unusual double-wave mean longitudinal magnetic field curve, which was repeatedly interpreted as a signature of an exceptionally strong, quadrupoledominated magnetic field topology.

Using a set of nearly one hundred Stokes $I$ and 52 Stokes $V$ high-resolution observations acquired for HR 5624 with the HARPSpol instrument at the ESO 3.6-m telescope, we find that the previously proposed quadrupolar field topology models are inconsistent with the observed polarisation profiles of metal lines. Interpretation of the LSD Stokes $V$ profiles with an 


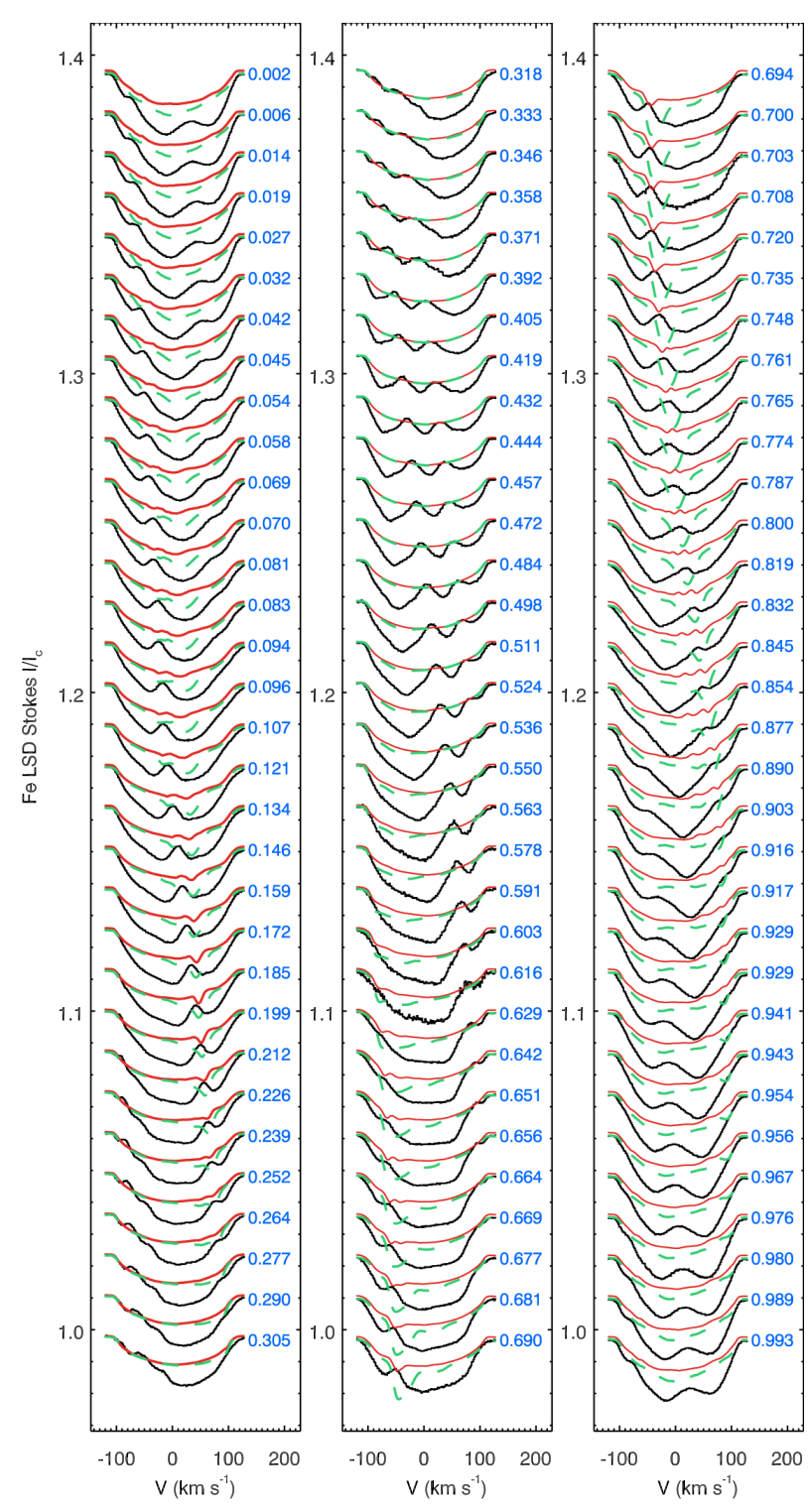

Fig. 18. Comparison of the observed Fe LSD Stokes $I$ profiles (black histogram) with calculations according to the diffusion theory predictions. The red solid lines show theoretical profiles for the iron distribution with $\log N_{\mathrm{Fe}} / N_{\mathrm{tot}}=-2.0$ in the regions with the local field inclination $\geq 75^{\circ}$ and $\log N_{\mathrm{Fe}} / N_{\mathrm{tot}}=-3.5$ elsewhere. The green dashed lines correspond to similar calculation with $\log N_{\mathrm{Fe}} / N_{\mathrm{tot}}=-2.0$ adopted for the surface regions with the field inclination $\geq 45^{\circ}$. Spectra corresponding to different rotation phases are offset vertically. Rotation phases are indicated to the right of each spectrum.

MDI code points to an asymmetric, bipolar field geometry with a large, weak positive field region and a smaller, strong negative field zone comprised of two distinct magnetic spots with a somewhat different field orientation. An independent MDI analysis of $\mathrm{Si}, \mathrm{Cr}$, and Fe lines yields consistent magnetic field maps, indicating the mean local field strength of 4.0-4.4 kG and the maximum local field of about $12 \mathrm{kG}$ at the negative magnetic pole. The field geometry is dominated by the dipolar component, which accounts for $65-71 \%$ of the total magnetic field energy. No evidence of a significant toroidal field is found in our magnetic mapping results.

The distorted, non-axisymmetric dipolar (rather than axisymmetric quadrupolar) magnetic field structure of HR 5624 qualitatively agrees with numerical simulations of fossil magnetic field evolution in stably stratified stellar interiors. Our results obtained for HR 5624 together with other recent MDI studies suggest that linear quadrupolar fields, often employed for describing Ap/Bp star magnetic field topologies, do not exist in real stars. Instead, the surface fossil field structure appears either as a (sometimes strongly distorted) dipole in the majority of stars or as a far more complex superposition of magnetic spots in a few others.

We have derived horizontal distributions of six chemical elements, including $\mathrm{Si}, \mathrm{Cr}, \mathrm{Fe}, \mathrm{Mg}$, Ti, and $\mathrm{Nd}$ using LSD Stokes profiles and intensity profiles of individual spectral lines. These abundance maps are characterised by high-contrast, large-scale overabundance features, which show no simple correlations with the local magnetic field strength and orientation. We showed that the observed $\mathrm{Fe}$ and $\mathrm{Cr}$ profile variation contradicts expectations of the current theoretical diffusion models, which predict narrow overabundance zones coinciding with the magnetic equator for the iron-peak and almost all other chemical elements. We suggest that this controversy should be resolved by making theoretical models more realistic and, in particular, by incorporating an anisotropic mass loss in diffusion modelling.

Acknowledgements. O.K. acknowledges financial support from the Knut and Alice Wallenberg Foundation, the Swedish Research Council, and the Swedish National Space Board. G.A.W. is supported by a Discovery Grant from the Natural Science and Engineering Research Council (NSERC) of Canada.

\section{References}

Alecian, E. 2014, in Putting A Stars into Context: Evolution, Environment, and Related Stars, Proc. Int. Conf. held in Moscow June 2013, eds. G. Mathys, E. R. Griffin, O. Kochukhov, R. Monier, \& G. M. Wahlgren, 84 Alecian, G. 2015, MNRAS, 454, 3143

Alecian, G., \& Stift, M. J. 2010, A\&A, 516, A53

Alecian, G., Stift, M. J., \& Dorfi, E. A. 2011, MNRAS, 418, 986

Aurière, M., Wade, G. A., Silvester, J., et al. 2007, A\&A, 475, 1053

Babel, J. 1992, A\&A, 258, 449

Babel, J., \& Montmerle, T. 1997, A\&A, 323, 121

Bagnulo, S., Landolfi, M., Mathys, G., \& Landi Degl'Innocenti, M. 2000, A\&A, 358,929

Bagnulo, S., Wade, G. A., Donati, J.-F., et al. 2001, A\&A, 369, 889

Bagnulo, S., Landi Degl'Innocenti, M., Landolfi, M., \& Mathys, G. 2002, A\&A, 394, 1023

Bagnulo, S., Landolfi, M., Landstreet, J. D., et al. 2009, PASP, 121, 993

Bailey, J. D. 2014, A\&A, 568, A38

Bailey, J. D., Grunhut, J., Shultz, M., et al. 2012, MNRAS, 423, 328

Borra, E. F., \& Landstreet, J. D. 1975, PASP, 87, 961

Braithwaite, J. 2008, MNRAS, 386, 1947

Braithwaite, J., \& Nordlund, A. 2006, A\&A, 450, 1077

Deutsch, A. J. 1958, in Electromagnetic Phenomena in Cosmical Physics, ed. B. Lehnert, IAU Symp., 6, 209

Donati, J.-F., \& Landstreet, J. D. 2009, ARA\&A, 47, 333

Donati, J.-F., Semel, M., Carter, B. D., Rees, D. E., \& Collier Cameron, A. 1997, MNRAS, 291, 658

Donati, J.-F., Howarth, I. D., Jardine, M. M., et al. 2006, MNRAS, 370, 629

Fares, R., Donati, J.-F., Moutou, C., et al. 2012, MNRAS, 423, 1006

Fossati, L., Castro, N., Schöller, M., et al. 2015, A\&A, 582, A45

Gayley, K. G., \& Owocki, S. P. 2014, in IAU Symp., eds. G. Meynet, C. Georgy, J. H. Groh, \& P. Stee, 307, 375

Hubrig, S., North, P., \& Schöller, M. 2007, Astron. Nachr., 328, 475

Kochukhov, O. 2015, A\&A, 580, A39

Kochukhov, O. 2016, in Cartography of the Sun and the Stars, Lect. Notes Phys., eds. J.-P. Rozelot, \& C. Neiner, 914 (Springer Int. Publishing), 177

Kochukhov, O. 2017, A\&A, 597, A58

Kochukhov, O., \& Bagnulo, S. 2006, A\&A, 450, 763

Kochukhov, O., \& Piskunov, N. 2002, A\&A, 388, 868

Kochukhov, O., \& Wade, G. A. 2010, A\&A, 513, A13

Kochukhov, O., \& Wade, G. A. 2016, A\&A, 586, A30

Kochukhov, O., Bagnulo, S., Wade, G. A., et al. 2004, A\&A, 414, 613

Kochukhov, O., Tsymbal, V., Ryabchikova, T., Makaganyk, V., \& Bagnulo, S. 2006, A\&A, 460, 831 
Kochukhov, O., Adelman, S. J., Gulliver, A. F., \& Piskunov, N. 2007, Nat. Phys., 3,526

Kochukhov, O., Makaganiuk, V., \& Piskunov, N. 2010, A\&A, 524, A5

Kochukhov, O., Lundin, A., Romanyuk, I., \& Kudryavtsev, D. 2011, ApJ, 726, 24

Kochukhov, O., Wade, G. A., \& Shulyak, D. 2012, MNRAS, 421, 3004

Kochukhov, O., Lüftinger, T., Neiner, C., Alecian, E., \& MiMeS Collaboration 2014, A\&A, 565, A83

Korhonen, H., González, J. F., Briquet, M., et al. 2013, A\&A, 553, A27

Krtička, J. 2014, A\&A, 564, A70

Landstreet, J. D. 1990, ApJ, 352, L5

Landstreet, J. D., \& Mathys, G. 2000, A\&A, 359, 213

Landstreet, J. D., Bagnulo, S., Andretta, V., et al. 2007, A\&A, 470, 685

LeBlanc, F., Monin, D., Hui-Bon-Hoa, A., \& Hauschildt, P. H. 2009, A\&A, 495, 937

Lehmann, H., Tkachenko, A., Fraga, L., Tsymbal, V., \& Mkrtichian, D. E. 2007, A\&A, 471, 941

Mathys, G. 2002, in Astrophysical Spectropolarimetry, eds. J. Trujillo-Bueno,

F. Moreno-Insertis, \& F. Sánchez, 101

Mathys, G. 2017, A\&A, 601, A14

Mathys, G., \& Hubrig, S. 1997, A\&AS, 124, 475

Mayor, M., Pepe, F., Queloz, D., et al. 2003, The Messenger, 114, 20

Michaud, C., Alecian, G., \& Richer, J. 2015, Atomic Diffusion in Stars (Springer)

Mikulášek, Z., Krtička, J., Henry, G. W., et al. 2011, A\&A, 534, L5

Moss, D. 2003, A\&A, 403, 693

Nesvacil, N., Shulyak, D., Ryabchikova, T. A., et al. 2013, A\&A, 552, A28

Netopil, M., Paunzen, E., Maitzen, H. M., North, P., \& Hubrig, S. 2008, A\&A, 491,545

Piskunov, N., \& Kochukhov, O. 2002, A\&A, 381, 736

Piskunov, N. E., \& Valenti, J. A. 2002, A\&A, 385, 1095

Piskunov, N., Snik, F., Dolgopolov, A., et al. 2011, The Messenger, 143, 7
Rusomarov, N., Kochukhov, O., Piskunov, N., et al. 2013, A\&A, 558, A8

Rusomarov, N., Kochukhov, O., Ryabchikova, T., \& Ilyin, I. 2016, A\&A, 588, A138

Ryabchikova, T. 2008, Contributions of the Astronomical Observatory Skalnate Pleso, 38, 257

Ryabchikova, T., Kochukhov, O., \& Bagnulo, S. 2008, A\&A, 480, 811

Ryabchikova, T., LeBlanc, F., \& Shulyak, D. 2011, in Magnetic Stars, eds. D. O. Kudryavtsev, \& I. I. Romanyuk, 69

Ryabchikova, T., Piskunov, N., Kurucz, R. L., et al. 2015, Phys. Sci., 90, 054005

Schneider, F. R. N., Podsiadlowski, P., Langer, N., Castro, N., \& Fossati, L. 2016, MNRAS, 457, 2355

Shulyak, D., Tsymbal, V., Ryabchikova, T., Stütz, C., \& Weiss, W. W. 2004, A\&A, 428, 993

Shulyak, D., Ryabchikova, T., Mashonkina, L., \& Kochukhov, O. 2009, A\&A, 499, 879

Silvester, J., Wade, G. A., Kochukhov, O., et al. 2012, MNRAS, 426, 1003

Silvester, J., Kochukhov, O., \& Wade, G. A. 2014, MNRAS, 440, 182

Snik, F., Kochukhov, O., Piskunov, N., et al. 2011, in Solar Polarization 6, Proc. Conf. held in Maui June 2010, eds. J. R. Kuhn et al., ASP Conf. Ser., 437, 237

Stibbs, D. W. N. 1950, MNRAS, 110, 395

Stift, M. J., \& Alecian, G. 2016, MNRAS, 457, 74

Thompson, I. B., \& Landstreet, J. D. 1985, ApJ, 289, L9

Townsend, R. H. D., Owocki, S. P., \& Groote, D. 2005, ApJ, 630, L81

Ud-Doula, A., Owocki, S. P., \& Townsend, R. H. D. 2009, MNRAS, 392, 1022

Unruh, Y. C., \& Collier Cameron, A. 1995, MNRAS, 273, 1

Urpin, V. 2016, Astron. Nachr., 337, 633

Wade, G. A., Grunhut, J. H., \& MiMeS Collaboration 2012, in Circumstellar Dynamics at High Resolution, eds. A. C. Carciofi, \& T. Rivinius, ASP Conf. Ser., 464, 405

Wolff, S. C. 1968, PASP, 80, 281 